\title{
On the dynamics of the four-dimensional rigid body in a quadratic potential field
}

\author{
Angel Zhivkov ${ }^{\text {a) }}$ \\ Differential Equations, Department of Mathematics and Informatics, Sofia University, \\ $5 \mathrm{~J}$. Bourchier, 1126 Sofia, Bulgaria \\ Milena Stanislavova ${ }^{\text {b) }}$ \\ Department of Mathematics, University of Architecture and Civil Engineering, \\ I Hr. Smirnenski Rlv., 1421 Sofia, Bulgaria
}

(Received 30 January 1995; accepted for publication 19 April 1995)

We study the nondegenerated solutions of the rotation of a four-dimensional rigid body in a quadratic potential field. This problem has 6 degrees of freedom. We obtain 143 topologically different solutions and explicit formulas in Prym theta-functions. (c) 1995 American Institute of Physics.

\section{INTRODUCTION}

In the last two decades several algebro-geometric constructions in connection with methods in explicitly solving nonlinear equations of mathematical physics were developed. ${ }^{1-4}$ In particular the role of Riemann surfaces, Abelian varieties, and theta-functions was found to be decisive in finding quasiperiodic solutions of the celebrated Korteweg-de Vries equation $u_{t}=u u_{x}+u_{x x x}$ for which explicit formulas for $u(x, t)$ in terms of Riemann theta-functions were found. After the Korteweg-de Vries equation this method, known now as the finite-gap integration, was successfully applied to other equations, e.g., the sine-Gordon equation, ${ }^{5}$ nonlinear Schrödinger equation, etc. Decisive in the method of the finite-gap integration is that all these equations admit a commutator representation

$$
\left[L-\frac{\partial}{\partial t}, M-\frac{\partial}{\partial x}\right]=0
$$

where $L=L(x, t, \lambda)$ and $M=M(x, t, \lambda)$ are polynomials in the parameter $\lambda$ (called the spectral parameter) with coefficients $(n \times n)$-matrices depending on $x$ and $t$.

In the case when $L$ and $M$ are operators independent of $x$, the above system becomes a system of ordinary differential equations

$$
\frac{d}{d t} M=[L, M]
$$

Then $(L, M)$ is called the "Lax pair."

Even the case of a first degree polynomial $L$, i.e., the case of a linear $(n \times n)$-matrix operator

$$
L(\lambda, t)=\lambda \sqrt{-1} C+\sqrt{-1} U,
$$

where $C=\operatorname{diag}\left(c_{1}, \ldots, c_{n}\right), c_{i} \neq c_{j}, U=\left(U_{i j}\right)_{i, j=1}^{n}$ is the $(n \times n)$-matrix, $U_{i i}=$ const $i=1, \ldots, n$ ( $U$ is called matrix potential) lead to interesting physical problems. These operators were studied in details by Dubrovin in Ref. 6.

The equation

a)Electronic mail: zhivkov@fmi.uni-sofia.bg

b)Electronic mail: acetra@bgcict.bitnet 


$$
\Gamma: \operatorname{det}(w \cdot 1-M(t, \lambda))=0
$$

defines an affine algebraic curve which does not depend on $t$. The compactification $\Gamma^{\prime}$ of $\Gamma$ is called a spectrum of the operator $L . \Gamma^{\prime}$ has singularities at the points $\infty_{1}, \infty_{2}, \ldots, \infty_{n}$, defined by the condition $\lambda\left(\infty_{i}\right)=\infty, i=1, \ldots, n$. Any two points $\infty_{i}$ and $\infty_{j} \in \Gamma^{\prime}$ are different because of the requirement $c_{i} \neq c_{j}$ if $i \neq j$ in Eq. (1), see Ref. 6. After a desingularization, $\Gamma^{\prime}$ becomes a compact Riemann surface $\hat{\Gamma}$.

We notice that the solutions obtained by finite-gap integration are complex ones. On the other hand the potentials $U(t)$ which appear in the natural physical problems have real symmetries and to determine them directly is not an easy problem. Instead one can make use of the geometric origin of the solutions, or more precisely to use that the Riemann surfaces corresponding to real solutions have real symmetries. The simplest symmetry is the self-adjointness of $L$, i.e., $U^{*}$ $=U,{ }^{*}$ is the hermitean conjugation. ${ }^{7}$ The case $n=2$ (containing nonlinear Schrödinger, sineGordon equation, etc.) is studied in Ref. 8. The common self-adjoint potentials are studied in Ref. 6; it turns out that the corresponding Riemann surface $\hat{\Gamma}$ is real, i.e., there exists an antiholomorphic involution $\tau_{1}: \hat{\Gamma} \rightarrow \hat{\Gamma}$, see Sec. $I$.

Another case of interest is the case of the real potential $U$, i.e., $U_{i j}(t) \in \mathbf{R}$ if $t \in \mathbf{R}, i, j$ $=1, \ldots, n$, a case studicd in Ref. 6 . Now on the corresponding Ricmann surface $\hat{\Gamma}$ there exists an antiholomorphic involution $\tau_{2}: \hat{\Gamma} \rightarrow \hat{\Gamma}$ with different properties from that of $\tau_{1}$, see Sec. I.

Natural problems lead also to potentials which are both self-adjoint and real, see Ref. 9. For example this situation appears in the problem of rigid body rotating about a fixed point, or in the problem of the geodesic flow on $\operatorname{so}(p)$.

The aim of this article is to study the solutions of one concrete Hamiltonian system: rotation of a four-dimensional rigid body around a fixed point in a quadratic potential field. In Ref. 10 Bogoyavlenskij proved that (in the $n$-dimensional case) this problem admits Lax-representation, is completely integrable, and has 6 degrees of freedom (in the $n$-dimensional case it has $n(n-1) / 2$ degrees of freedom). The solutions of that problem are self-adjoint and real. The conditions of realness of the case $n=3$ were studied in Ref. 11 . We will prove that, in the case $n=4$, there are 143 topologically different solutions.

We. need to explain to the reader the term "topologically different solutions." In accordance with the classical Liouville-Arnold's theorem ${ }^{12}$ the compact invariant varieties of the completely integrable system for almost all values of the first integrals are homeomorphic with several $n$-dimensional tori. The solutions of the Hamiltonian system are straight linear over these tori. For a description of the topological nature of the Hamiltonian system we need to determine the topological type of the compact invariant varieties, after which we explain how the invariant compact varieties pass over from one to another, when the initial conditions are changed. Thus, we obtain the phase picture without some bifurcation's set.

We briefly describe the main results contained in the article. In the Sec. I we give the equations, representing the above problem and the corresponding Riemann surface $\Gamma$ together with the conditions of realness and self-adjointness. Because of the symmetries there exist antiholomorphic involutions $\tau_{1}: \Gamma \rightarrow \Gamma$ and $\tau_{2}: \Gamma \rightarrow \Gamma, \tau_{i}^{2}=$ identity over $\Gamma$ which have fixed properties. To solve the problem it is necessary to make a topological classification of the four-tuples $\left(\Gamma, \tau_{1}, \tau_{2}, \lambda\right)$ of the Riemann surfaces $\Gamma$ with two anti-involutions and 4-sheeted meromorphic function $\lambda$ : $\Gamma \rightarrow C P .{ }^{1}$ In Sec. II we find that there exist 36 topologically different four-tuples $\left(\Gamma, \tau_{1}, \tau_{2}, \lambda\right)$ and we compute the number of the invariant tori on each component. The general number of the invariant tori is 143 . This is the content of Theorem 1. In Sec. III we construct an appropriate basis in the one-dimensional homologies $H_{1}(\Gamma)$, in which the Riemann theta-function reduces to the Prym theta-function. Using this special basis we compute explicitly the tori, forming the Prym variety (on which the motion is straight linear), receive the explicit formulas for the angular velocities, and reduce the formulas in the Prym theta-functions over the Riemann surfaces of genus 6 and 3. We find the real symmetries of the values, participating in the formulas. We do 
this in one of the 36 cases only, but the formulas and the symmetries are analogous in the other cases. Theorem 2 in Sec. III contains this result.

\section{THE SPECTRUM OF THE PROBLEM}

Consider the problem of rotation of a four-dimensional rigid body around a fixed point in a quadratic potential field. The system, describing this motion is

$$
\begin{gathered}
\dot{M}=[M, W]-[U, I], \\
\dot{U}=[U, W],
\end{gathered}
$$

where $M=\left(M_{i, j}\right)_{i, j=1}^{4}, W=\left(W_{i, j}\right)_{i, j=1}^{4}$ are skew-symmetric, $I=\operatorname{diag}\left(I_{1}, I_{2}, I_{3}, I_{4}\right), I_{k}, k=1, \ldots, 4$ are real, positive, and different constants, $M_{i, j}=I_{k} I_{l} W_{i, j}, i, j, k, l$ are different indexes from 1 to 4 , $U=U^{t}=\left(U_{i, j}\right)_{i, j=1}^{4}$.

In Ref. 10 it has been proved that the problem of a $n$-dimensional rigid body, rotating around a fixed point in a quadratic potential field is completely integrable. In order to integrate Eq. (2) we use Lax-representation with spectral parameter $\lambda$

$$
\frac{d}{d t} L=[L, Q]
$$

where $L=\lambda^{2} \operatorname{diag}\left(I_{2} I_{3} I_{4}, I_{1} I_{3} I_{4}, I_{1} I_{2} I_{4}, I_{1} I_{2} I_{3}\right)+\lambda M+U, Q=W-\lambda I$. The matrices $M, W$, and $U$ are real.

The equation

$$
\Gamma: \operatorname{det}(L(t, \lambda)-y \cdot \mathbf{1})=0, \quad y \in \mathbf{C}
$$

defines an affine algebraic curve which does not depend on $t$. After compactification and desingularization, $\Gamma$ becomes a compact Riemann surface, called the spectrum of the problem. $\Gamma$ is a 4-sheeted covering of the complex line $\lambda$. The genus of $\Gamma$ is equal to 9 . Obviously there is a holomorphic involution $\sigma(y, \lambda)=(y,-\lambda)$ acting on $\Gamma$. The " $\sigma$-points" (points $x \in \Gamma$, satisfying $\sigma x=x)$ are 8 -these are $\lambda^{-1}(0)$ and $\lambda^{-1}(\infty)$. So, by the Riemann-Hurvitz theorem, the genus of $\Gamma_{1}=\Gamma / \sigma$ is equal to 3 .

Because of the real symmetries of the matrices $M, U$, and $W$ the operator $L(\lambda)$ is selfadjointed, i.e., $L^{*}(\lambda)=L(-\bar{\lambda})$ (here ${ }^{*}$ is the hermitean conjugation). Hence there exists an antiholomorphic involution $\tau_{1}: \Gamma \rightarrow \Gamma, \tau_{1}^{2}=$ identity, $\tau_{1}(y, \lambda)=(\bar{y},-\bar{\lambda})$ such that ${ }^{6}$

(1) The set $\Gamma^{\tau_{1}}=\left\{x \in \Gamma: \tau_{1} x=x\right\}$ separates $\Gamma$ into two components $\Gamma^{+}$and $\Gamma^{-} ; \tau_{1} \infty_{k}=\infty_{k}$, $k=1, \ldots, 4$.

(2) The function $\lambda$ does not have branch points over $\mathbf{i R P}^{1}=\{\sqrt{-1} z \mid z \in \mathbf{R} \cup \infty\}$ and $\lambda^{-1}\left(\mathbf{i R P}^{1}\right)=\Gamma^{\tau_{1}}$.

The operator $L(\lambda)$ is also "real," i.e., $\overline{L(\lambda)}=L(\bar{\lambda})$. Then there exists a second antiholomorphic involution $\tau_{2}: \Gamma \rightarrow \Gamma, \tau_{2}(y, \lambda)=(\bar{y}, \bar{\lambda})$ such that ${ }^{6}$

(1) $\tau_{2}$ commutes with $\tau_{1}\left(\tau_{1} \cdot \tau_{2}=\tau_{2} \cdot \tau_{1}\right)$.

(2) $\tau_{2} \infty_{i}=\infty_{i}, \quad i=1, \ldots, 4$.

According to the classical Liouville's theorem, ${ }^{12}$ for almost all values of the first integrals, the corresponding invariant compact varieties of a completely integrable system are homeomorphic with several $n$-dimensional tori, on which the motion is straight linear. For the computation of the tori where the flow of the system is linear we need to describe the structure of the surface $\Gamma$ with the above-mentioned symmetries. We do this in the next section. 


\section{TOPOLOGY OF THE SOLUTIONS}

Our aim is to make a topological classification of the four-tuples $\left(\Gamma, \tau_{1}, \tau_{2}, \lambda\right)$, where $\Gamma$ is a Riemann surface of genus $g=9$;

$\tau_{1}: \Gamma \rightarrow \Gamma$ is an anti-involution separating $\Gamma$, i.e., $\Gamma=\Gamma^{+} \cup \Gamma^{\tau_{1}} \cup \Gamma^{-}$;

$\Gamma^{\tau_{1}}=\left\{x \in \Gamma: \tau_{1} x=x\right\} ; \tau_{1} \Gamma^{+}=\Gamma^{-} ; \Gamma^{\tau_{1}}=\partial \Gamma^{+}=\partial \Gamma^{-}, \Gamma^{+} \cap \Gamma^{-}=\varnothing, \tau_{1}^{*} \lambda=-\bar{\lambda}, \Gamma^{\tau_{1}}$ $=\lambda^{-1}\left(\mathbf{i R P} \mathbf{P}^{1}\right)$;

$\tau_{2}: \Gamma \rightarrow \Gamma$ is an anti-involution, commuting with $\tau_{1}, \tau_{1} \tau_{2}=\tau_{2} \tau_{1}=\sigma$ and satisfying the conditions: $\tau_{2}^{*}=\bar{\lambda}$ and $\lambda^{-1}(0 \cup \infty) \subset \Gamma^{\tau_{2}}$

$\lambda: \Gamma \rightarrow C P^{\mathrm{I}}$ is a 4-sheeted meromorphic function.

The four-tuples $\left(\Gamma, \tau_{1}, \tau_{2}, \lambda\right)$ and $\left(\Gamma^{\prime}, \tau_{1}^{\prime}, \tau_{2}^{\prime}, \lambda^{\prime}\right)$ are called algebraically equivalent if there exists a biholomorphic map $f: \Gamma \rightarrow \Gamma^{\prime}$ such that the following diagrams are commutative:
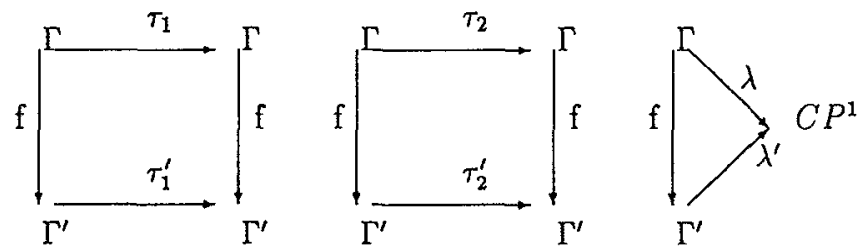

Let $H_{4}$ be the space of the above-mentioned classes of equivalence of four-tuples $\left(\Gamma, \tau_{1}, \tau_{2}, \lambda\right)$. Consider the space $H_{2}$ of pairs $(\Gamma, \lambda)$, forgetting temporarily the anti-involutions $\tau_{1}, \tau_{2} . \Gamma$ is a compact Riemann surface of genus $9, \lambda$ is a 4-sheeted meromorphic function without any branch points at $\infty$, so that $\lambda$ has 24 branch points over $\Gamma$, giving an account of multiplicities.

According to the classical Clebsch theorem ${ }^{13}$ the pair $(\Gamma, \lambda)$ can be expressed as a multivalued meromorphic function. Take 4 copies $S_{1}, \ldots, S_{4}$ of $C P^{1}$ and let $\lambda_{i}=\lambda_{\mid S_{i}}: S_{i} \rightarrow C P^{1}$ are biholomorphic maps, $i=1, \ldots, 4$. Take 12 disjoint paths $\gamma_{1}, \ldots, \gamma_{12}$ over $C P^{1}$ and give every $\gamma_{i}$ two indexes $\alpha_{i}, \beta_{i}, 1 \leqslant \alpha_{i}<\beta_{i} \leqslant 4$. Cut $S_{\alpha_{i}}$ and $S_{\beta_{i}}$ along $\lambda_{\alpha_{i}}^{-1}\left(\gamma_{i}\right)$ and $\lambda_{\beta_{i}}^{-1}\left(\gamma_{i}\right)$; glue the left beach of $\lambda_{\alpha_{i}}^{-1}\left(\gamma_{i}\right)$ with the right beach of $\lambda_{\beta_{i}}^{-1}\left(\gamma_{i}\right)$ and the right beach of $\lambda_{\alpha_{i}}^{-1}\left(\gamma_{i}\right)$ with the left beach of $\lambda_{\beta_{i}}^{-1}\left(\gamma_{i}\right)$. So we constructed the pair $(\Gamma, \lambda)$.

The choice of the paths $\gamma_{1}, \ldots, \gamma_{12}$ is inessential. If we replace $\gamma_{i}$ with $\tilde{\gamma}_{i}$ and $U \subset C P^{1}$ is the

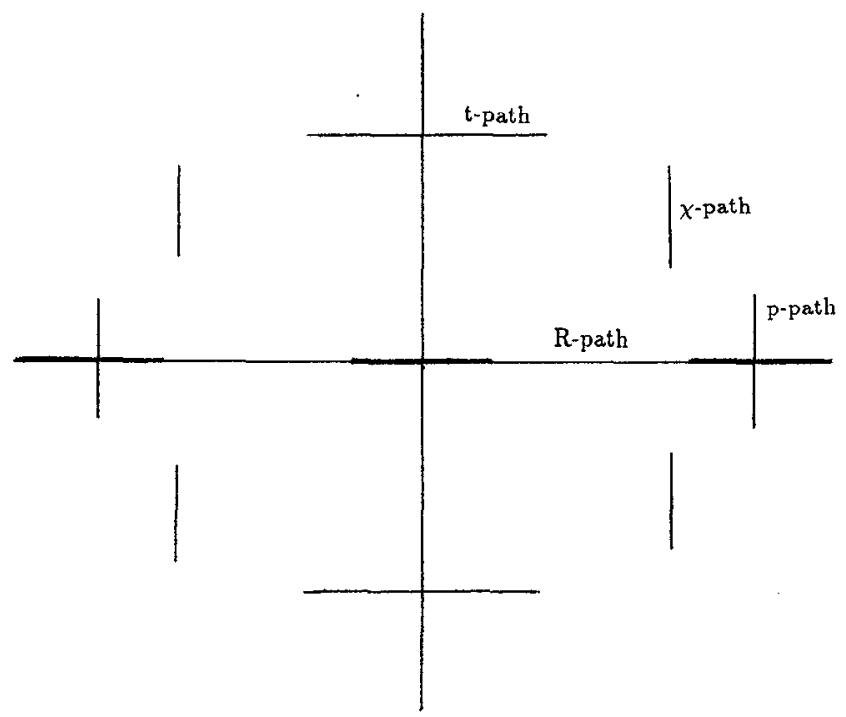

FIG. 1. Four types of paths. 
area between $\gamma_{i}$ and $\tilde{\gamma}_{i}$ we must change the indexes $\alpha_{j}$ and $\beta_{j}$, if $\gamma_{i} \subset U$. If $\alpha_{k}=\alpha_{i}$ then $\alpha_{k}$ becomes $\beta_{i}$. If $\alpha_{k}=\beta_{i}$ then $\alpha_{k}$ becomes $\alpha_{i}$. If $\beta_{k}=\beta_{i}$ then $\beta_{k}$ becomes $\alpha_{i}$.

Now, let us define a topology in the space $H_{2}$ of pairs $(\Gamma, \lambda)$. Two pairs $(\Gamma, \lambda)$ and $\left(\Gamma^{\prime}, \lambda^{\prime}\right)$ are called algebraically equivalent if there exists a biholomorphic map $f: \Gamma \rightarrow \Gamma^{\prime}$ such that $\lambda^{\prime}(f(x))=\lambda(x)$ for all $x \in \Gamma$. Then we consider that $(\Gamma, \lambda)=\left(\Gamma^{\prime}, \lambda^{\prime}\right)$ in $H_{2}$.

Continuous deformation or simply deformation of $(\Gamma, \lambda)$ in $\mathrm{H}_{2}$ we define as a continuous deformation of the paths $\gamma_{i}$ over $C P^{1}$ (including deformations of the ends of $\gamma_{i}$ ), without changing the indexes $\alpha_{i}, \beta_{i}$. This defines topology in $H_{2}$.

The space $\mathrm{H}_{4}$ of four-tuples inherits the topology from $\mathrm{H}_{2}$. Every component $\mathrm{K}$ of $\mathrm{H}_{4}$ has a representative $\left(\Gamma^{\prime}, \tau_{1}^{\prime}, \tau_{2}^{\prime}, \lambda^{\prime}\right) \in K$ which can be expressed as in the Clebsch theorem but symmetrically about $\mathbf{R}$ and iR paths $\gamma_{i}$. More precisely, $\left(\Gamma^{\prime}, \tau_{1}^{\prime}, \tau_{2}^{\prime}, \lambda^{\prime}\right)$ has four types of paths:

R-paths: Pairs of paths $\left(\gamma_{i}, \bar{\gamma}_{i}\right) \subset \mathbf{R}$, symmetrical about iR.

p-paths: Pairs of paths $\left(\gamma_{j}, \bar{\gamma}_{j}\right) ; \gamma_{j}, \bar{\gamma}_{j}$ cross $\mathbf{R}$ and are symmetrical about $\mathbf{i R}$.

t-paths: Pairs of paths $\left(\gamma_{k}, \bar{\gamma}_{k}\right) ; \gamma_{k}, \bar{\gamma}_{k}$ cross iR and are symmetrical about $\mathbf{R}$.

$\boldsymbol{\chi}$-paths: Four paths $\left(\gamma_{l}, \bar{x}, \gamma_{l}^{\prime}, \bar{\gamma}^{\prime}\right), \gamma_{l} \cap \mathbf{R}=\varnothing, \gamma_{l} \cap \mathbf{i R}=\varnothing$, symmetrical about $\mathbf{R}$ and $\mathbf{i R}$.

Moreover the symmetrical paths have the same indexes $\left(\alpha_{k} \beta_{k}\right)$, see Fig. 1 . The $R-, p-, t-$, and $\chi$-paths with indexes $(\alpha \beta)$ we note by $(\alpha \beta)_{R},(\alpha \beta)_{p},(\alpha \beta)_{t}$, and $(\alpha \beta)_{\chi}$ correspondingly.
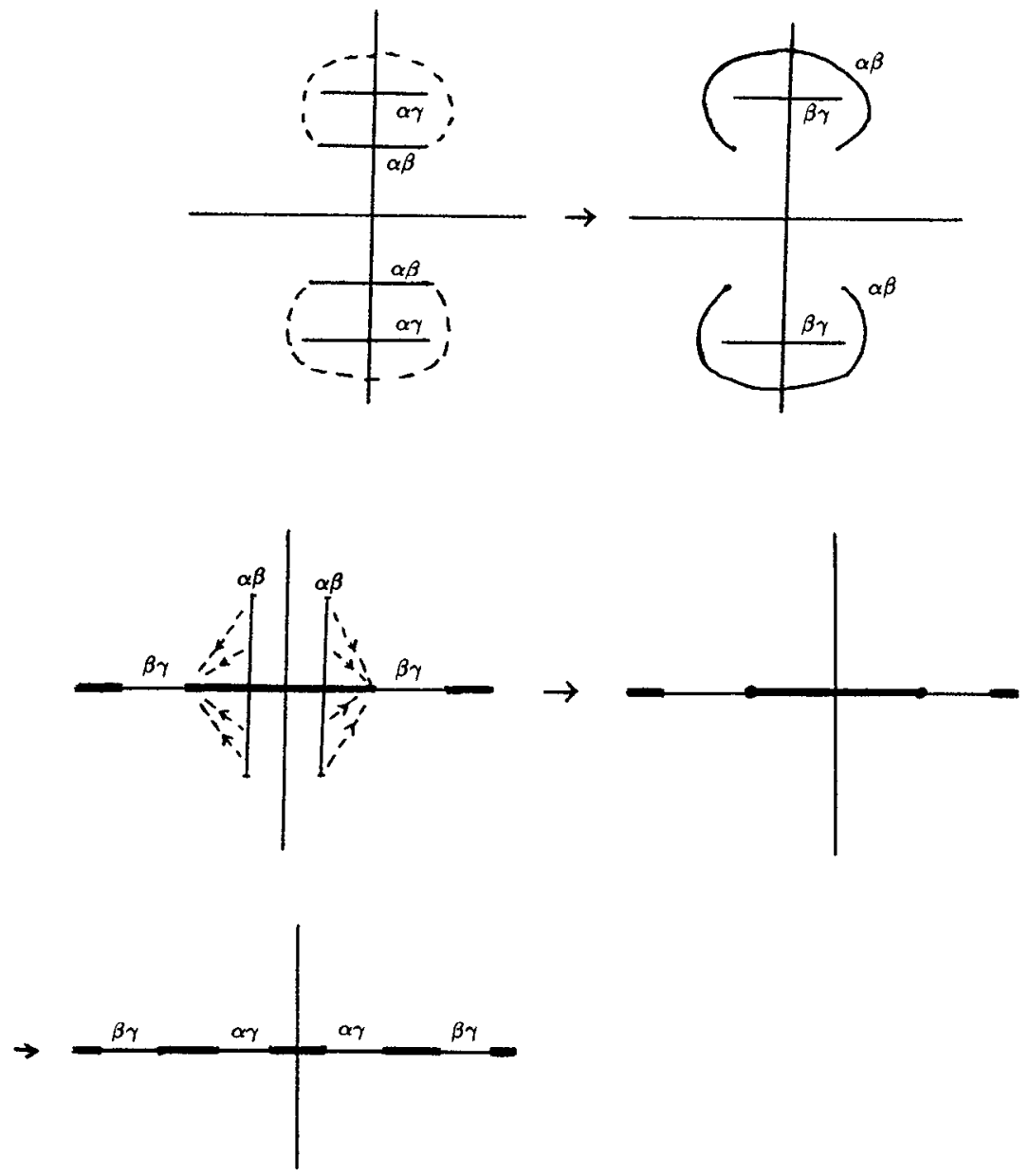

FIG. 2. Operation $O_{1}(\alpha \gamma, \alpha \beta \rightarrow \alpha \beta, \beta \gamma)$. 


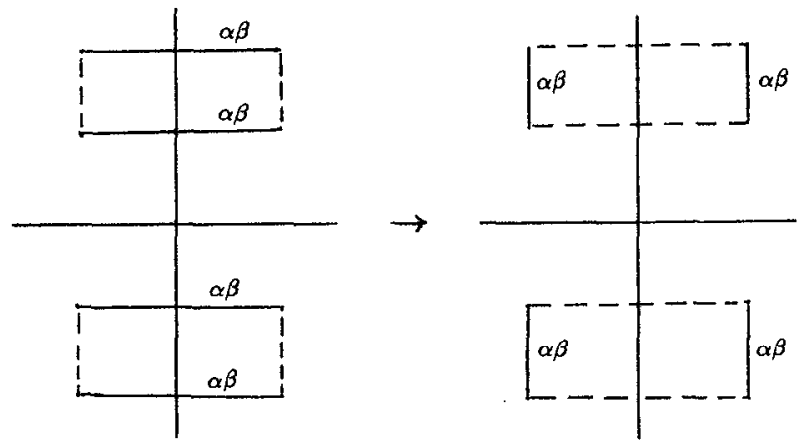

FIG. 3. Operation $\mathrm{O}_{2}(\alpha \beta)$.

Recall that the our aim is to put every four-tuple $\left(\Gamma, \tau_{1}, \tau_{2}, \lambda\right) \in H_{4}$ in "standard type," i.e., on every component $V \subset H_{4}$ we choose an element $v \in V$ and then deform $V$ to $v$. For this purpose we consider the following 6 operations, which are deformations on $\mathrm{H}_{4}$.

Operation 1. Replacement of a path $\xi$, having indexes $(\alpha \beta)$ with a path $\xi^{\prime}$ with the same ends and the same indexes $(\alpha \beta)$; change the indexes $\alpha$ with $\beta$ and the indexes $\beta$ with $\alpha$ in the area $G$ between $\xi$ and $\xi^{\prime}$. All that is done symmetrically about $\mathbf{R}$ and $\mathbf{i R}$ and has 5 versions-for $p$-, $t$-, and $\chi$-paths, for $\chi$ - and $t$-paths, and for $p$ - and $\chi$-paths. We note this operation by $O_{1}$ $(\alpha \gamma, \alpha \beta \rightarrow \alpha \beta, \beta \gamma)$. See Fig. 2 .

Operation 2. Replacement of two neighboring $t$-paths, having the same indexes $(\alpha \beta)$ with two $\chi$-paths, having indexes $(\alpha \beta)$, too. We note this operation with $O_{2}(\alpha \beta)$, see Fig. 3 .

Operation 3. Replacement of the $t$-path, having indexes $(\alpha \gamma)$ with $t$-path, having indexes $(\beta \delta)$ provided that there are two $R$-paths with indexes $(\alpha \beta)$ and $(\gamma \delta)$. We note this operation with $\mathrm{O}_{3}$ $(\alpha \gamma \rightarrow \beta \delta)$, see Fig. 4 .

Operation 4. Permutation of the numbers of sheets of the covering $-\{1,2,3,4\} \rightarrow\left\{i_{1}, i_{2}, i_{3}, i_{4}\right\}$. We note this operation with $O_{4}\left(i_{1}, i_{2}, i_{3}, i_{4}\right)$.

Operation 5. Replacement of the $\chi$-path, having indexes $(\alpha \gamma)$ with the $\chi$-path having indexes $(\beta \delta)$, provided there are two $R$-paths, having indexes $(\alpha \beta)$ and $(\gamma \delta)$. We note this operation with $O_{5}(\alpha \gamma \rightarrow \beta \delta)$, see Fig. 5 .

Operation 6. Replacement of two $p$-paths $\xi, \xi^{\prime}$, having indexes $(\alpha \beta)$ with two $t$-paths $\zeta, \zeta^{\prime}$, having the same ends and the same indexes $(\alpha \beta)$, but in the area $G$, between $\xi, \xi^{\prime}, \zeta, \zeta^{\prime}$ changing the indexes $\alpha$ with $\beta$ and changing the indexes $\beta$ with $\alpha$. We note this operation by $O_{6}$, see Fig. 6 .

Lemma 1: Given a set of $t$-paths, it can be changed and ordered in the following form: $\left(\alpha_{1} \beta_{1}\right),\left(\alpha_{1} \beta_{2}\right), \ldots,\left(\alpha_{1} \beta_{k_{1}}\right) ;\left(\alpha_{2} \gamma_{1}\right),\left(\alpha_{2} \gamma_{2}\right), \ldots,\left(\alpha_{2} \gamma_{k_{2}}\right), \ldots$, where $\alpha_{i} \neq \alpha_{j}, \beta_{i} \neq \beta_{j}, \gamma_{i} \neq \gamma_{j}$ for $i \neq j$.

Proof: We use only $O_{1}(\alpha \gamma, \alpha \beta \rightarrow \alpha \beta, \beta \gamma)$ and $O_{2}(\alpha \beta)$ if necessary.

Lemma 2: If there are at least two $R$-paths with indexes (12) and (34) then there exists such deformation in $H_{4}$, which transforms $R$-paths so that there is one $R$-path, having indexes (34) and the others $R$-paths have indexes (12).

Proof: Let us transform three $R$-paths with indexes (12), (12), and (34) to three $R$-paths with indexes (12), (34), and (34). Moreover the other paths will not change their indexes. As the Riemann surface is connected there exists a $t$-path or a $R$-path, having indexes (12), (14), (23), or (24). We consider only the case when there is $t$-path with indexes (13). We use only $O_{1}(\alpha \gamma, \alpha \beta \rightarrow \alpha \beta, \beta \gamma)$ and $O_{6}$ if necessary and the stages of the deformation are: 

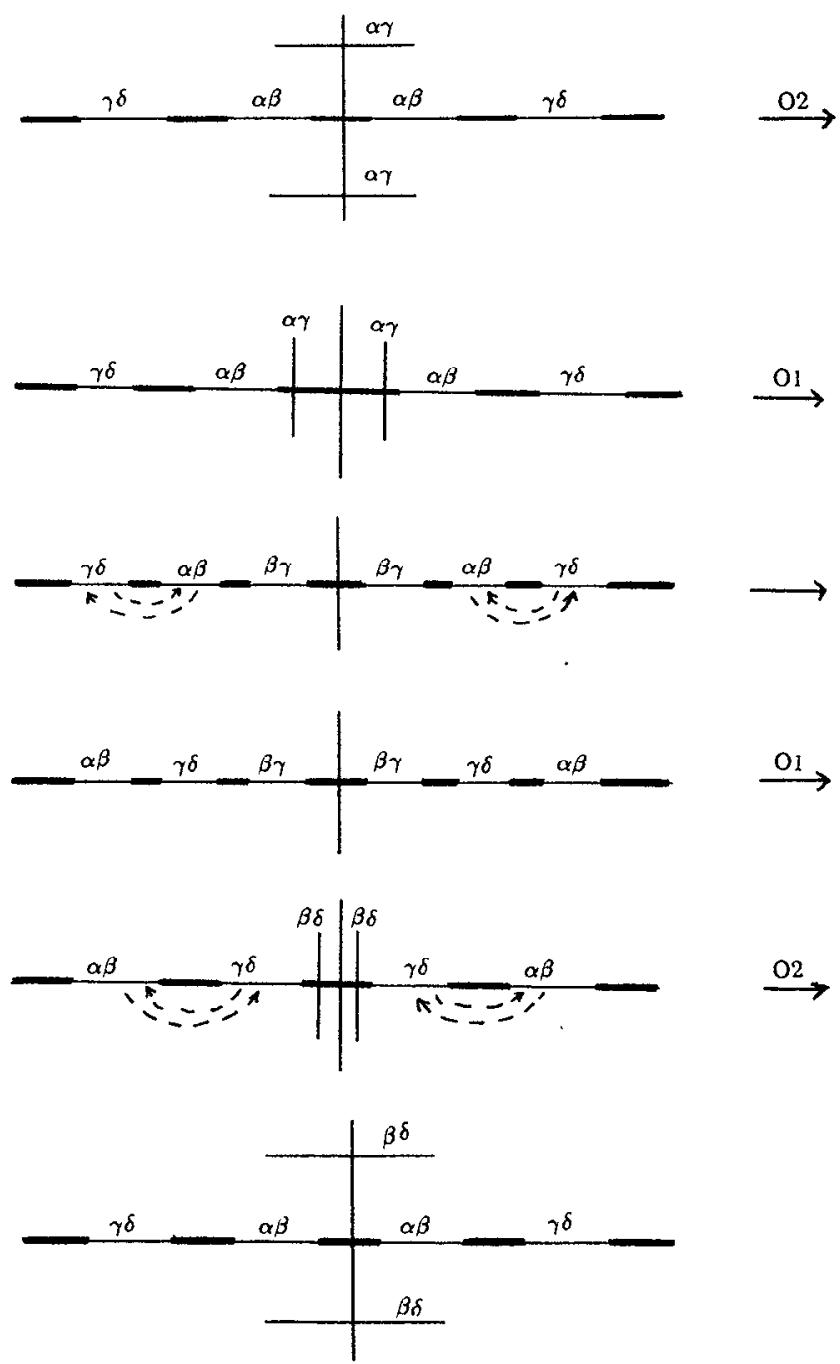

FIG. 4. Operation $O_{3}(\alpha \gamma \rightarrow \beta \delta)$.

$$
\begin{gathered}
(12)_{R},(12)_{R},(34)_{R},(13)_{t} \rightarrow(23)_{R},(23)_{R},(13)_{p},(34)_{R} \rightarrow(23)_{R},(23)_{R},(34)_{R},(14)_{R} \rightarrow \\
(23)_{R},(14)_{R},(24)_{p},(34)_{R} \rightarrow(14)_{R},(24)_{p},(34)_{R},(34)_{R} \rightarrow(14)_{R},(34)_{R},(34)_{R},(23)_{R} \rightarrow \\
(23)_{R},(13)_{p},(34)_{R},(34)_{R} \rightarrow(12)_{R},(34)_{R},(34)_{R},(13)_{t} .
\end{gathered}
$$

We call the sheets $\alpha$ and $\beta$ of the covering "connected with t-path" if there exists a sequence of $t$-paths with indexes $\left(\alpha \alpha_{1}\right),\left(\alpha_{1} \alpha_{2}\right), \ldots,\left(\alpha_{k} \beta\right)$. This relation separates the sheets $1,2,3,4$ from the disjoint $t$-components. If there is not a $t$-path with an index $\alpha$ we assume that the sheet $\alpha$ is a single $t$-component.

Using operation 6 we transform the $p$-paths into $t$-paths. Applying Lemma 1 and operation 4 we can make the indexes of $t$-paths of the first $t$-component to be $(12),(13), \ldots,\left(1 k_{1}\right)$, of the second $t$-component $\left(i_{1}, i_{1}+1\right),\left(i_{1}, i_{1}+2\right), \ldots,\left(i_{1}, k_{2}\right)$, etc. Thus we obtain a minimal number of $t$-paths, for which there are not two $t$-paths, having the same indexes and moreover sequence of indexes of 

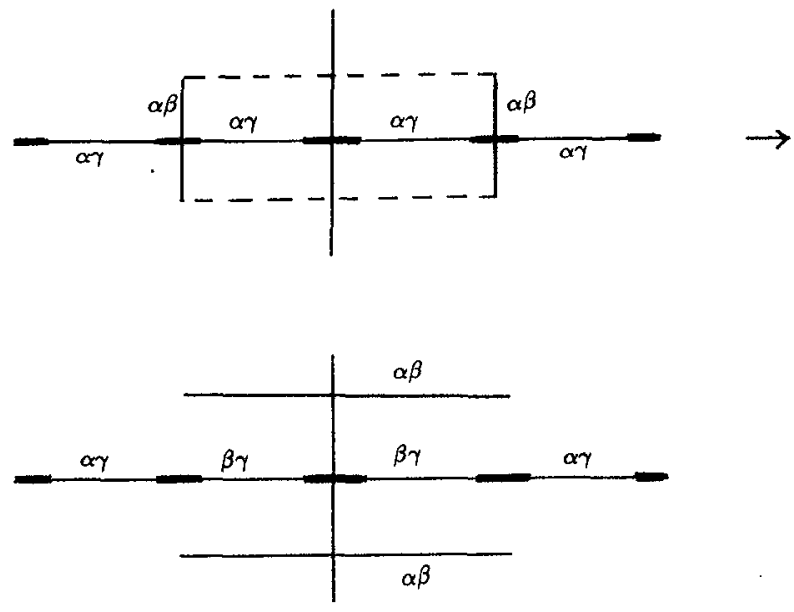

FIG. 5. Operation $O_{5}(\alpha \gamma \rightarrow \beta \delta)$.

$t$-paths is minimal in the lexicographical ordering. If the $R$-paths also are minimal in number, there are not two $R$-paths with indexes $(\alpha \beta),(\alpha \gamma), \beta \neq \gamma$ and applying Lemma 2 we receive the minimal in the lexicographical ordering sequence of indexes of $R$-paths. Using operation 1 we receive the minimal in the lexicographical ordering sequence of indexes of $\chi$-paths.
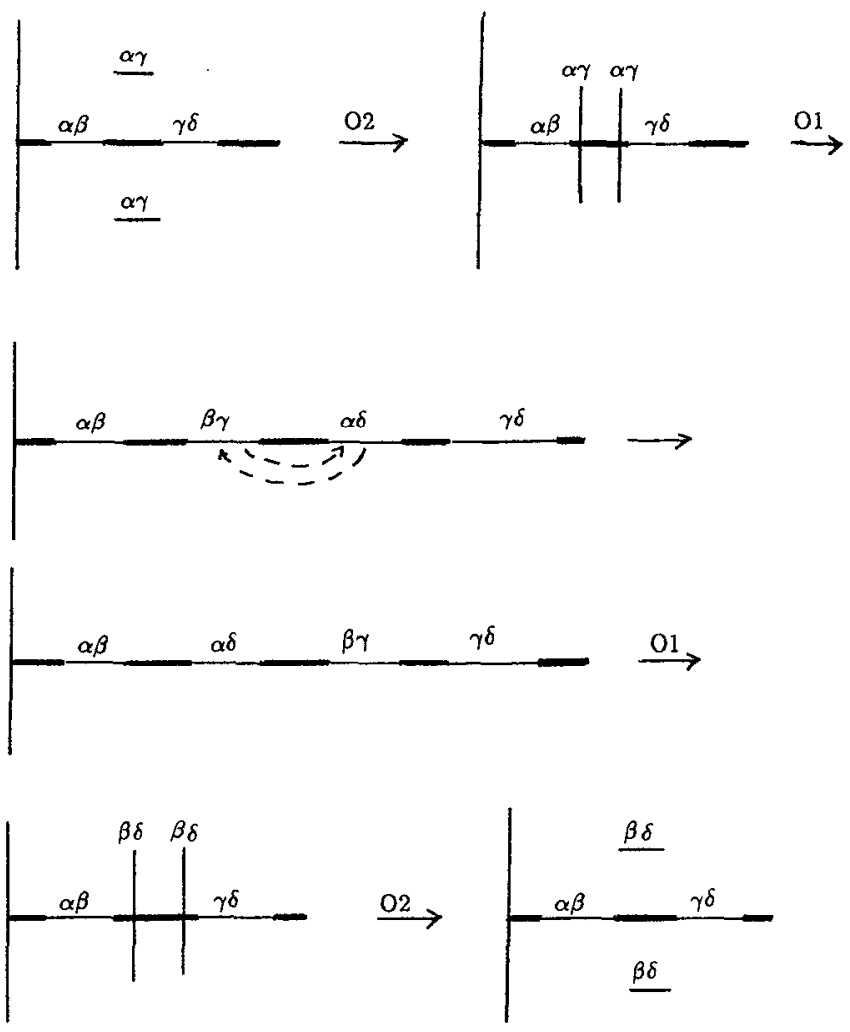

FIG. 6. Operation $\mathrm{O}_{6}$. 
TABLE I. Possibilities for the $t$-paths.

\begin{tabular}{|c|c|c|c|c|}
\hline \multicolumn{5}{|c|}{$\begin{array}{l}\text { number of connected components } \rightarrow \\
\downarrow \text { number of } t \text {-paths }\end{array}$} \\
\hline Number & 1 & 2 & 3 & 4 \\
\hline 0 & 1 & 1 & 1 & $\varnothing$ \\
\hline 1 & 1 & 1 & (12) & 1 \\
\hline 2 & 1 & $(12),(34)$ or $(12),(13)$ & 1 & 1 \\
\hline 3 & $(12),(13),(14)$ & 1 & 1 & 1 \\
\hline
\end{tabular}

We define standard type of an element of $\mathrm{H}_{4}$ as such representative of the same component of $H_{4}$, for which: (a) there exist only $t-, R$-, and $\chi$-paths, (b) the number of $R$-paths is minimal, (c) the number of $t$-paths is minimal, (d) the sequence of indexes of paths $(t|R| \chi)$ is minimal in the lexicographical ordering.

According to Lemma 1, all possibilities for the $t$-paths are as in Table I. All possibilities for the $\chi$-paths, according to the number and indexes of $t$-paths, are given in Tables II-V together with the operations, by which one couple of indexes replace the other when it is possible and when we like it. The empty squares in the tables are the possible combinations.

For the $t$-paths there exist the following five cases.

Case I. There is not a $t$-path.

Then all possible couples of indexes of $\chi$-paths are (12), (13), (14), (23), (24), and (34). Combinations of two $t$-paths are given in Table II. If there are three $\chi$-paths, there are not $R$ - and $t$-paths, because of the number of branch points. Hence $\chi$-paths have indexes (12), (13), and (14) in order to have the connected Riemann surface.

Case II. There is one $t$-path (with indexes (12)).

Applying operation 1 we replace with $\chi$-paths, having indexes (23) or (24) with $\chi$-paths, having indexes (13) or (14), respectively. The possibilities are given in Table III by the empty squares.

Case III. There are two $t$-paths with indexcs (12) and (13) correspondingly.

Applying operation 1 we replace (23), (24), and (34) indexes of $\chi$-paths, with (13), (14), and (14), respectively. See in Table IV the possibilities for combinations of two $\chi$-paths.

Case IV. There are two $t$-paths with indexes (12) and (34) correspondingly.

Applying operation 1 we replace (23), (24), and (14) indexes of $\chi$-paths with (23), (24), and (34), respectively. See table V.

Case V. There are three $t$-paths with indexes (12), (13), and (14).

By operation 1 we replace every $\chi$-path with a $\chi$-path, having indexes (12).

The above five cases are all combinations of $t$-paths. In Table VI are given all possible combinations of indexes of $R$-paths and $t$-paths together with the operations, which replace indexes when it is possible and when we like it. All combinations of $R-, t$-, and $\chi$-paths are given in Tables VII-XI. If there is not such element of $H_{4}$ (because there are not enough branch points or if the obtained Riemann surface is unconnected) the corresponding square is crossed out. The

TABLE II. Possibilities for the $t$-paths.

\begin{tabular}{lcccccc}
\hline \hline Indexes & $(12)$ & $(13)$ & $(14)$ & $(23)$ & $(24)$ & $(34)$ \\
\hline$(12)$ & & \multicolumn{1}{c}{$o_{1}(12,23 \rightarrow 12,13)$} & $o_{1}(12,24 \rightarrow 12,14)$ \\
$(13)$ & 1 & & & $o_{1}(13,23 \rightarrow 12,13)$ & & $o_{1}(13,34 \rightarrow 13,14)$ \\
$(14)$ & 1 & 1 & & $o_{1}(14,24 \rightarrow 12,14)$ & & $o_{1}(14,34 \rightarrow 13,14)$ \\
$(23)$ & 1 & 1 & 1 & & & $o_{1}(23,24 \rightarrow 23,24)$ \\
$(24)$ & 1 & 1 & 1 & 1 & & $o_{1}(24,34 \rightarrow 23,24)$ \\
$(34)$ & 1 & 1 & 1 & 1 & 1 & \\
\hline
\end{tabular}


TABLE III. Possibilities for the $\chi$-paths.

\begin{tabular}{lcccc}
\hline Indexes & $(12)$ & $(13)$ & $(14)$ & $(34)$ \\
\hline$(12)$ & & & & \\
$(13)$ & 1 & & & $o_{1}(13,34 \rightarrow 13,14)$ \\
$(14)$ & 1 & 1 & & $o_{1}(14,34 \rightarrow 13,14)$ \\
$(34)$ & 1 & 1 & 1 & $0^{2}$ \\
\hline
\end{tabular}

operations, by which elements of one and the same component of $H_{4}$ are changed with the element of this component which is in standard type, are given in the tables too. Therefore the empty squares in Tables VII-XI correspond to different components of $H_{4}$. The different components of $H_{4}$ are noted by $\mathbf{A}, \mathbf{B 1}, \mathbf{B} 2, \ldots, \mathbf{Q} 1, \mathbf{Q} 2$.

We call the connected components of $\Gamma^{\tau_{i}}=\left\{x \in \Gamma: \tau_{i} x=x\right\} \tau_{i}$-ovals. Each oval is a cycle over $\Gamma$. According to Ref. 14, the set $\Gamma^{\tau_{1}} \cup \Gamma^{\tau_{2}}$ consists of the following admissable elements:

(1) $\tau_{2}$-ovals $k$ and $\tau_{1} k$, where $k \cap \tau_{1} k=\varnothing$, see Fig. 7 .

(2) Sequence of ovals $k_{1}, \ldots, k_{2 s}, s \geqslant 1, k_{2 i} \subset \Gamma^{\tau_{2}}, k_{2 i-1} \subset \Gamma^{\tau_{1}}, i=1, \ldots, s$ and the sections $k_{1} \cap k_{2}, k_{2} \cap k_{3}, \ldots, k_{2 i-1} \cap k_{2 i}, k_{2 i} \cap k_{1}$ consist of one point. Such components of $\Gamma^{\tau_{1}} \cup \Gamma^{\tau_{2}}$ are called garlands. See Fig. 8.

Define the signature deg $\lambda_{/ k}$ for the $\tau_{2}$-oval $k$ as the power of the covering $\lambda: k \rightarrow \mathbf{R P}^{1}$. If $k$ is the $\tau_{2}$-oval we define deg $\lambda_{/ k}$ as the power of the covering $\lambda: k \rightarrow \mathbf{R} \mathbf{P}^{1}$. Moreover when the antiinvolution $\tau$ separates $\Gamma, \Gamma^{+}$induces an orientation over $\Gamma^{\tau}$. Thus we define the sign of the number $\operatorname{deg} \lambda_{/ k}$ when the anti-involution separates $\Gamma{ }^{15}$ According to Ref. 15, the admissable four-tuples $\left(\Gamma, \tau_{1}, \tau_{2}, \lambda\right)$ have the following topological invariants:

(1) $c$-the number of garlands and deg $\lambda_{/ k}$ for every oval $k$ of anti-involution $\tau_{2}$ included in this garland (the signatures of $\lambda$ over ovals of $\tau_{1}$ are always equal to 1 );

(2) $L_{2}$-the half of the $\tau_{2}$-ovals, out of garlands (without " $\sigma$-points");

(3) $\epsilon_{2}$-the type of $\tau_{2}: \epsilon_{2}=1$ if $\Gamma^{\tau_{2}}$ does not separate $\Gamma$ and $\epsilon_{2}=2$ otherwise. Moreover, if $\epsilon_{2}=2$ and if the unequality

$$
\left|\sum_{k_{i}-\tau_{2}-\text { oval }} \lambda / k_{i}\right|<\sum_{k_{i}-\tau_{2}-\text { oval }}\left|\lambda / k_{i}\right|=2
$$

is fulfilled, there exists an additional invariant $\chi_{2}:{ }^{14}$

(4) $\chi_{2}$ is the genus of the surface

$$
\Gamma^{+} \cap \lambda^{-1}\{z \in \mathbf{C} \mid \operatorname{Im} z>0\}, \quad \chi_{2}+L_{2} \leqslant 3 .
$$

In our case, $\chi_{2}=0$ or $\chi_{2}=2$, because $\chi_{2}$ is even.

The above four invariants completely determine the different components of the space $\mathrm{H}_{4}$. Thus we obtain the following

TABLE IV. Possibilities for the $\chi$-paths.

\begin{tabular}{cccc}
\hline \hline Indexes & $(12)$ & $(13)$ & $(14)$ \\
\hline$(12)$ & 1 & & \\
$(13)$ & 1 & 1 \\
$(14)$ & 1 & \\
\hline
\end{tabular}


TABLE V. Possibilities for the $\chi$-paths.

\begin{tabular}{cccc}
\hline \hline Indexes & $(12)$ & $(13)$ & $(34)$ \\
\hline$(12)$ & & & \\
$(13)$ & 1 & 1 \\
$(34)$ & 1 & 1 \\
\hline
\end{tabular}

Theorem 1. There exist 36 topologically different four-tuples $\left(\Gamma, \tau_{1}, \tau_{2}, \lambda\right)$, which can be the spectra for the problem (2), i.e., the space $\mathrm{H}_{4}$ consists of 36 components. Representatives of these four-tuples are pictured in Fig. 9 where the $\tau_{2}$-ovals of degree 0 over $R P^{1}$ are drawn bold. The number of the invariant tori in every case is $2^{n_{i}}$. This number $n_{i}$ and the values of the invariants are given in Table XII. The general number of the invariant tori is 143 .

Proof: It is seen from Tables I-XI that every component of $\mathrm{H}_{4}$ can be transformed to one of these 36 representatives. Since they have different values of at least one invariant, they are different. The number of the invariant tori is computed by the following

Theorem. (Reference 16) The set $T=\left\{z_{0}: \tau_{1} z_{0}=-z_{0}, \tau_{2} z_{0}=z_{0}+\tau_{2} \Delta-\Delta(\bmod \Lambda)\right\} \subset J(\Gamma)$ consists of $2^{m-l}$ elements, where $m=c+L_{2}, c$ is the number of garlands, $2 L_{2}$ is the number of ovals of $\tau_{2}$ out of garlands, $\Delta$ is the Riemann theta-divisor, $\Lambda$ is the lattice of the periods. All $2^{m-1}$ components have dimension (over $R) \frac{l}{2}(g+\eta-1)$ where $2 \eta$ is the number of $\sigma$-points on $\Gamma$.

TABLE VI. Combinations of $\boldsymbol{R}$ - and $\boldsymbol{t}$-paths.

\begin{tabular}{|c|c|c|c|c|c|}
\hline \multicolumn{6}{|c|}{$\begin{array}{l}\text { indexes of } t \text {-paths } \rightarrow \\
\downarrow \text { indexes of } R \text {-paths }\end{array}$} \\
\hline$\varnothing$ & & 1 & & & 1 \\
\hline \multicolumn{6}{|l|}{ (12) } \\
\hline (13) & $o_{4}(1324)+$ & & $\begin{array}{c}o_{4}(1324)+ \\
o_{1}(23,12 \rightarrow 12,13)\end{array}$ & & \\
\hline (14) & $O_{4}(1432)$ & $o_{4}(1243)$ & & $o_{4}(1243)$ & $\begin{array}{c}o_{4}(2341)+ \\
o_{1}(24,12 \rightarrow 12,14)+ \\
o_{1}(23,12 \rightarrow 12,13)\end{array}$ \\
\hline (23) & $o_{4}(3214)$ & $o_{4}(2134)$ & $\begin{array}{c}o_{4}(3124)+ \\
o_{1}(13,23 \rightarrow 12,13)\end{array}$ & $o_{4}(2134)$ & $\begin{array}{c}o_{4}(4123)+ \\
o_{1}(14,24 \longrightarrow 12,14)+ \\
o_{1}(14,34 \rightarrow 13,14)\end{array}$ \\
\hline (24) & $o_{4}(4231)$ & $o_{4}(2143)$ & $\begin{array}{c}o_{4}(3124)+ \\
o_{1}(13,23 \rightarrow 12,13)\end{array}$ & $o_{4}(2143)$ & $\begin{array}{c}o_{4}(4123)+ \\
o_{1}(14,24 \rightarrow 12,14)+ \\
o_{1}(14,34 \rightarrow 13,14)\end{array}$ \\
\hline (34) & $o_{4}(3412)$ & & $\begin{array}{c}o_{4}(2314)+ \\
o_{1}(23,12 \rightarrow 12,13)\end{array}$ & $o_{4}(3412)$ & $\begin{array}{c}o_{4}(3412)+ \\
o_{1}(34,13 \rightarrow 13,14)\end{array}$ \\
\hline \multicolumn{6}{|l|}{$(12),(34)$} \\
\hline$(13),(24)$ & $o_{4}(1324)$ & & $\begin{array}{c}o_{4}(2314)+ \\
o_{1}(23,12 \rightarrow 12,13)\end{array}$ & & \\
\hline$(14),(23)$ & $o_{4}(3412)$ & $o_{\Delta}(2134)$ & $\begin{array}{c}o_{4}(3124)+ \\
o_{1}(13,23 \rightarrow 12,13)\end{array}$ & $o_{4}(2134)$ & $\begin{array}{c}o_{4}(2341)+ \\
o_{1}(24,12 \rightarrow 12,14)+ \\
o_{1}(23,12 \rightarrow 13,12)\end{array}$ \\
\hline
\end{tabular}


TABLE VII. Combinations of $R$ - and $\chi$-paths, when there are not $t$-paths.

\begin{tabular}{|c|c|c|c|}
\hline $\begin{array}{l}\text { indexes of } R \text {-paths } \rightarrow \\
\downarrow \text { indexes of } \chi \text {-paths } \\
\text { Indexes }\end{array}$ & $\varnothing$ & (12) & $(12),(34)$ \\
\hline$\varnothing$ & 1 & 1 & 1 \\
\hline (12) & 1 & 1 & 1 \\
\hline (13) & 1 & 1 & C1 \\
\hline (14) & 1 & 1 & $o_{4}(1243)$ \\
\hline (23) & $\backslash$ & 1 & $a_{4}(2134)$ \\
\hline (24) & 1 & 1 & $o_{4}(2143)$ \\
\hline (34) & 1 & 1 & 1 \\
\hline$(12),(12)$ & 1 & $\backslash$ & 1 \\
\hline$(13),(13)$ & 1 & 1 & C2 \\
\hline$(14),(14)$ & 1 & 1 & $o_{4}(1243)$ \\
\hline$(23),(23)$ & 1 & 1 & $o_{4}(1243)$ \\
\hline$(24),(24)$ & 1 & 1 & $o_{4}(2143)$ \\
\hline$(34),(34)$ & 1 & 1 & 1 \\
\hline$(12),(13)$ & 1 & 1 & $\mathrm{C3}$ \\
\hline$(12),(14)$ & 1 & 1 & $o_{4}(1243)$ \\
\hline$(12),(34)$ & 1 & 1 & 1 \\
\hline$(13),(14)$ & 1 & B1 & $\begin{array}{c}o_{4}(3412)+ \\
o_{1}(13,23 \rightarrow 12,13)\end{array}$ \\
\hline$(13),(24)$ & 1 & B2 & $o_{5}(24 \rightarrow 13)$ \\
\hline$(14),(23)$ & 1 & $o_{4}(2134)$ & $o_{4}(2134)$ \\
\hline$(14),(23)$ & 1 & $o_{4}(2134)$ & $\mathrm{O}_{4}(2134)$ \\
\hline$(12),(13),(14)$ & $\mathbf{A}$ & 11 & III \\
\hline
\end{tabular}

\section{EXPLICIT SOLUTIONS IN PRYM THETA-FUNCTIONS}

In Ref. 6 formulas for the components $W_{i j}$ of the angular velocity have been found. Let a canonical basis $(a, b)=\left(a_{1}, \ldots, a_{9}, b_{1}, \ldots, b_{9}\right)$ in the one-dimensional homologies $H_{1}(\Gamma)$ be fixed. Let $A: \Gamma \rightarrow J(\Gamma)$ be the Abel's map onto the Jacobian $J(\Gamma)$ of $\Gamma$ with an initial point $x_{0} \in \Gamma$; $A_{j s}=A\left(\infty_{j}-\infty_{s}\right)$, where $A: \Gamma \rightarrow C^{9}$ is the continued Abel's map after paths $\gamma_{1}, \ldots, \gamma_{4}$ from $x_{0}$ to

TABLE VIII. Combinations of $R$ - and $\chi$-paths, when there is one $t$-path with indexes (12).

\begin{tabular}{|c|c|c|c|c|c|}
\hline $\begin{array}{c}\text { indexes of } R \text {-paths } \rightarrow \\
\downarrow \text { indexes of } \chi \text {-paths } \\
\text { Indexes }\end{array}$ & (12) & (13) & (34) & $\begin{array}{c}(1,2),(34) \\
\quad+(12)\end{array}$ & $\begin{array}{c}(1,3),(24) \\
\quad+(13)\end{array}$ \\
\hline$\varnothing$ & 1 & 1 & 1 & 1 & H1 \\
\hline (12) & 1 & 1 & 1 & 1 & $\mathbf{H} \mathbf{2}$ \\
\hline (13) & 1 & 1 & F1 & $\mathbf{G}$ & $\mathrm{H3}$ \\
\hline (14) & 1 & $\mathbf{E} 1$ & $o_{4}(1234)$ & $o_{4}(1234)$ & $\begin{array}{c}o_{5}(14 \rightarrow 23)+ \\
o_{1}(23 \rightarrow 13)\end{array}$ \\
\hline (34) & 1 & $\mathbf{E 2}$ & 1 & 1 & $o_{5}(34 \rightarrow 12)+$ \\
\hline$(12),(12)$ & 1 & 1 & 1 & * & * \\
\hline$(13),(13)$ & 1 & 1 & $\begin{array}{c}o_{1}(13 \rightarrow 23)+ \\
o_{1}(23,13 \rightarrow 13,12)\end{array}$ & * & $\star$ \\
\hline$(14),(14)$ & 1 & 1 & $o_{4}(1243)$ & $\star$ & * \\
\hline$(34),(34)$ & 1 & $\mathbf{E 3}$ & 1 & * & $\star$ \\
\hline$(12),(13)$ & 1 & 1 & $\mathbf{F 2}$ & * & * \\
\hline$(12),(14)$ & 1 & $\mathbf{E 4}$ & 1 & * & * \\
\hline$(12),(34)$ & 1 & $\mathbf{E 5}$ & 1 & $\star$ & $\star$ \\
\hline$(13),(14)$ & D & E6 & F3 & * & $\star$ \\
\hline
\end{tabular}


TABLE IX. Combinations of $R$ - and $\chi$-paths, when there are two $t$-paths with indexes (12),(13).

\begin{tabular}{|c|c|c|c|c|}
\hline $\begin{array}{l}\text { indexes o } \\
\downarrow \text { indexes } \\
\text { Indexes }\end{array}$ & $\varnothing$ & $(12),(12)$ & $(14),(14)$ & $(12),(34)$ \\
\hline$\varnothing$ & 1 & 1 & K1 & $\mathbf{L 1}$ \\
\hline (12) & 1 & 1 & $\mathbf{K} 2$ & L2 \\
\hline (13) & 1 & 1 & $o_{4}(1324)$ & $\begin{array}{l}o_{1}(12,13 \rightarrow 12,23)+ \\
o_{1}(13,23 \rightarrow 12,23)+ \\
o_{1}(12,23 \rightarrow 12,13)+\end{array}$ \\
\hline (14) & $\star$ & $\mathbf{J}$ & $\mathbf{K 3}$ & $\begin{array}{c}o_{5}(14 \rightarrow 23)+ \\
o_{1}(23,13 \rightarrow 12,13)\end{array}$ \\
\hline$(12),(12)$ & 1 & * & * & $\star$ \\
\hline (13),(13) & 1 & $\star$ & $\star$ & * \\
\hline$(14),(14)$ & $\begin{array}{c}o_{1}(12,23 \rightarrow 12,14)+ \\
o_{1}(14,12 \rightarrow 12,24)\end{array}$ & $\star$ & $\star$ & * \\
\hline$(12),(13)$ & 1 & * & * & $\star$ \\
\hline$(12),(14)$ & I & $\star$ & * & $\star$ \\
\hline$(13),(14)$ & $o_{1}(12,23 \rightarrow 12,13)$ & $\star$ & $\star$ & $\star$ \\
\hline
\end{tabular}

$\infty_{1}, \ldots, \infty_{4}$ have been fixed. Let $\Omega_{i}$ be the meromorphic differential with a pole in $\infty_{i}$, the principal part of which is $\sqrt{-1} d \lambda$ and with zero $a$-periods; $U^{i} \in \mathbf{C}^{9}$ is the vector of $b$-periods of $\Omega_{i}$. Let $\epsilon\left(\infty_{i}, \infty_{j}\right)=E\left(\infty_{i}, \infty_{j}\right)\left[d \lambda\left(\infty_{i}\right) d \lambda\left(\infty_{j}\right)\right]^{-1 / 2}$ where $E(x, y), x, y \in \Gamma$ is the prime-form of the Riemann surface $\Gamma$ with a local coordinate $\lambda^{-1}$ around $\infty_{k} \cdot{ }^{17}$ Let $B$ be the Riemann matrix of $\Gamma$ and $\Theta\left[\begin{array}{l}\alpha \\ \beta\end{array}\right](z \mid B)$ be the Riemann theta-function with characteristics $\alpha, \beta, \Theta\left[{ }_{0}^{0}\right](z \mid B)=\Theta(z \mid B)$. Then ${ }^{16}$

$$
W_{j s}(t)=\left(I_{j}-I_{s}\right) \frac{\mu_{j}}{\mu_{s}} \exp \left(t \sum_{i=1}^{4} I_{i} \int_{\infty_{s}}^{x_{j}} \Omega_{i}+\left\langle\varphi\left(z_{0}\right), A_{j s}\right\rangle\right) \frac{\Theta\left(A_{j s}+z_{0}+t U\right)}{\Theta\left(z_{0}+t U\right) \epsilon\left(\infty_{j}, \infty_{s}\right)},
$$

where $z_{0}=A\left(d-\infty_{1}-\cdots-\infty_{4}-\Delta\right), \quad \Delta \quad$ is the Riemann theta-divisor, $U=I_{1} U^{1}+I_{2} U^{2}+I_{3} U^{3}+I_{4} U^{4}$ and the integrals are considered as a principal value if necessary. The basis $(a, b)=\left(a_{1}, \ldots, a_{9}, b_{1}, \ldots, b_{9}\right)$ in $H_{1}(\Gamma)$ is called the $\sigma$-basis if

TABLE X. Combinations of $R$ - and $\chi$-paths, when there are two $t$-paths with indexes (12),(34).

\begin{tabular}{|c|c|c|c|c|c|}
\hline \multicolumn{6}{|c|}{$\begin{array}{l}\text { indexes of } R \text {-paths } \rightarrow \\
\downarrow \text { indexes of } \chi \text {-paths }\end{array}$} \\
\hline$\varnothing$ & 1 & 1 & 01 & 1 & $o_{3}(34 \rightarrow 12)$ \\
\hline (12) & 1 & 1 & $\mathbf{0 2}$ & 1 & $o_{3}(34 \rightarrow 12)$ \\
\hline (13) & * & $\mathbf{N}$ & 03 & $o_{3}(34 \rightarrow 12)$ & $o_{3}(34 \rightarrow 12)$ \\
\hline (34) & 1 & 1 & $\mathrm{O}_{4}(3412)$ & * & $O_{5}(34 \rightarrow 12)$ \\
\hline$(12),(12)$ & 1 & * & $\star$ & $\star$ & $\star$ \\
\hline$(13),(13)$ & $\begin{array}{c}o_{1}(13,12 \rightarrow 12,23)+ \\
o_{1}(13,23 \rightarrow 12,13)\end{array}$ & $\star$ & * & * & * \\
\hline$(34),(34)$ & 1 & * & $\star$ & * & * \\
\hline (12).(13) & $\mathbf{M}$ & 夫 & $\star$ & * & $\star$ \\
\hline$(12),(34)$ & 1 & * & $\star$ & $\star$ & * \\
\hline$(13),(34)$ & $\begin{array}{c}o_{1}(14,34 \rightarrow 13,34)+ \\
o_{7}(13,34 \rightarrow 13,14)\end{array}$ & * & $\star$ & $\star$ & * \\
\hline
\end{tabular}


TABLE XI. Combinations of $R$ - and $\chi$-paths, when there are three $t$-paths with indexes (12),(13),(14).

\begin{tabular}{lcccc}
\hline \hline $\begin{array}{l}\text { indexes of } R \text {-paths } \rightarrow \\
\downarrow \text { indexes of } \chi \text {-paths } \\
\text { Indexes }\end{array}$ & $(12)$ & $(13)$ & $(12),(12),(34)$ & $(13),(13),(24)$ \\
\hline$\varnothing$ & P1 & Q1 & $o_{3}(14 \rightarrow 23)$ & $\star$ \\
$(12)$ & P2 & Q2 & $o_{3}(14 \rightarrow 23)$ \\
$\star$
\end{tabular}

$$
\begin{gathered}
\sigma\left(a_{1}\right)=-a_{7}, \sigma\left(a_{2}\right)=-a_{8}, \sigma\left(a_{3}\right)=-a_{9}, \sigma\left(a_{4}\right)=-a_{4}, \sigma\left(a_{5}\right)=-a_{5}, \sigma\left(a_{6}\right)=-a_{6}, \\
\sigma\left(a_{7}\right)=-a_{1}, \sigma\left(a_{8}\right)=-a_{2}, \sigma\left(a_{9}\right)=-a_{3}, \sigma\left(b_{1}\right)=-b_{7}, \sigma\left(b_{2}\right)=-b_{8}, \\
\sigma\left(b_{3}\right)=-b_{9}, \sigma\left(b_{4}\right)=-a_{4}, \sigma\left(b_{5}\right)=-b_{5}, \sigma\left(b_{6}\right)=-b_{6}, \\
\sigma\left(b_{7}\right)=-b_{1}, \sigma\left(b_{8}\right)=-b_{2}, \sigma\left(b_{9}\right)=-b_{3} .
\end{gathered}
$$

In a $\sigma$-basis the theta-function $\Theta\left(z_{1}, \ldots, z_{9} \mid B\right)$ of genus 9 can be reduced to theta-functions of genus 6 and 3 . According to ${ }^{17}$

$$
\begin{aligned}
\Theta= & \left(z_{1}, \ldots, z_{9} \mid B\right)=\Theta\left(z_{1}+z_{7}, z_{2}+z_{8}, z_{3}+z_{9}, z_{4}, z_{5}, z_{6} \mid 2 \Pi\right) \Theta\left(z_{1}-z_{7}, z_{2}-z_{8}, z_{3}-z_{9} \mid 2 \zeta\right) \\
& +\Theta\left[\begin{array}{cccccc}
\frac{1}{2} & \frac{1}{2} & \frac{1}{2} & 0 & 0 & 0 \\
0 & 0 & 0 & 0 & 0 & 0
\end{array}\right]\left(z_{1}+z_{7}, z_{2}+z_{8}, z_{3}+z_{9}, z_{4}, z_{5}, z_{6} \mid 2 \Pi\right) \\
& \times \Theta\left[\begin{array}{ccc}
\frac{1}{2} & \frac{1}{2} & \frac{1}{2} \\
0 & 0 & 0
\end{array}\right]\left(z_{1}-z_{7}, z_{2}-z_{8}, z_{3}-z_{9} \mid 2 \zeta\right),
\end{aligned}
$$

where $B$ is the Riemann matrix of $\Gamma, \Pi=\left(\Pi_{i j}\right)_{i, j=1}^{6}$ is the Prym matrix, $\zeta=\left(\zeta_{i j}\right)_{i, j=1}^{3}$ is the Riemann matrix of $\Gamma_{1}=\Gamma / \sigma\left(g\left(\Gamma_{1}\right)=3\right) . B, \Pi$, and $\zeta$ are related by

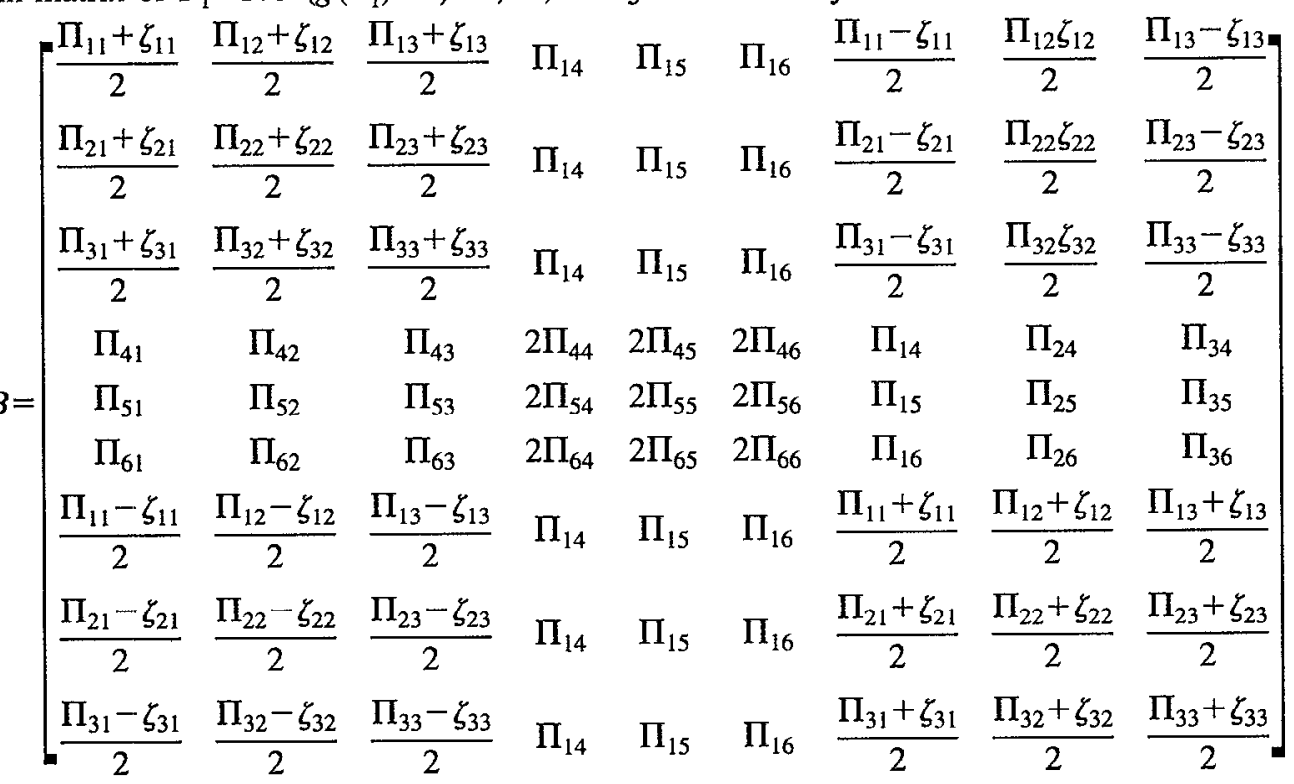


TABLE XII. Theorem 1 .

\begin{tabular}{|c|c|c|c|c|c|}
\hline Case & Indexes of the paths $(t|R| \chi)$ & Garlands & $L_{2}$ & $\epsilon_{2}$ & Tori \\
\hline 1 & 2 & 3 & 4 & 5 & 6 \\
\hline $\mathbf{A}$ & $(\varnothing|\varnothing| 12,13,14)$ & $\begin{array}{l}G_{1}(1,1) ; G_{2}(1,1) \\
G_{3}(1,1) ; G_{4}(1,1)\end{array}$ & 0 & 2 & 8 \\
\hline BI & $(\varnothing|12,12| 13,14)$ & $\begin{array}{c}G_{1}(1,0,1,0) \\
G_{2}(1,1) ; G_{3}(1,1)\end{array}$ & 1 & 2 & 8 \\
\hline $\mathbf{B 2}$ & $(\varnothing|12,12| 13,24)$ & $\begin{array}{c}G_{1}(1,0,1,0) \\
G_{2}(1,1) ; G_{3}(1,-1)\end{array}$ & 1 & 2 & 8 \\
\hline $\mathbf{C 1}$ & $(\varnothing|12,12,12,34| 13)$ & $\begin{array}{l}G_{1}(1,0,1,0) \\
G_{2}(1,0,1,0)\end{array}$ & 2 & 2 & 8 \\
\hline $\mathrm{C2}$ & $(\varnothing|12,34| 13,13)$ & $\begin{array}{l}G_{1}(1,0,1,0) \\
G_{2}(1,0,1,0)\end{array}$ & 0 & 2 & 2 \\
\hline $\mathbf{C 3}$ & $(\varnothing|12,34| 12,13)$ & $\begin{array}{l}G_{1}(1,0,1,0) \\
G_{2}(1,0,1,0)\end{array}$ & 0 & 1 & 2 \\
\hline $\mathbf{D}$ & $(12|12| 13,14)$ & $\begin{array}{c}G_{1}(1,0,1,0) \\
G_{2}(1,1) ; G_{3}(1,1)\end{array}$ & 0 & 1 & 4 \\
\hline E1 & $(12|13,13,13| 14)$ & $\begin{array}{c}G_{1}(1,1,1,0,1,0) \\
G_{2}(1,1)\end{array}$ & 2 & 2 & 8 \\
\hline E2 & $(12|13,13,13| 34)$ & $\begin{array}{c}G_{1}(1,1,1,0,1,0) \\
G_{2}(1,-1)\end{array}$ & 2 & 2 & 8 \\
\hline $\mathbf{E 3}$ & $(12|13| 34,34)$ & $\begin{array}{c}G_{1}(1,1,1,0,1,0) \\
G_{2}(1,-1)\end{array}$ & 0 & $\begin{array}{c}2 \\
x_{2}=2\end{array}$ & 2 \\
\hline E4 & $(12|13| 12,14)$ & $\begin{array}{c}G_{1}(1,1,1,0,1,0) \\
G_{2}(1,1)\end{array}$ & 0 & 2 & 2 \\
\hline $\mathbf{E 5}$ & $(12|13| 12,34)$ & $\begin{array}{c}G_{1}(1,1,1,0,1,0) \\
G_{2}(1,-1)\end{array}$ & 0 & $\begin{array}{c}2 \\
\chi_{2}=0\end{array}$ & 2 \\
\hline E6 & $(12|13| 13,14)$ & $\begin{array}{c}G_{1}(1,1,1,0,1,0) \\
G_{2}(1,1)\end{array}$ & 0 & 1 & 2 \\
\hline F1 & $(12|34,34,34| 13)$ & $\begin{array}{l}G_{1}(1,1,1,1) \\
G_{2}(1,0,1,0)\end{array}$ & 2 & 2 & 8 \\
\hline $\mathbf{F} 2$ & $(12|34| 13,13)$ & $\begin{array}{l}G_{1}(1,1,1,1) \\
G_{2}(1,0,1,0)\end{array}$ & 0 & 2 & 2 \\
\hline $\mathbf{F 3}$ & $(12|34| 13,14)$ & $\begin{array}{l}G_{1}(1,1,1,1) \\
G_{2}(1,0,1,0)\end{array}$ & 0 & 1 & 2 \\
\hline $\mathbf{G}$ & $(12|12,12,34| 13)$ & $\begin{array}{c}G_{1}(1,0,1,0) \\
G_{2}(1,0,1,0)\end{array}$ & 1 & 1 & 4 \\
\hline $\begin{array}{l}\text { H1 } \\
\text { 21 }\end{array}$ & $(12|13,13,13,13,24| \varnothing)$ & $G_{1}(1,0,1,0,1,0,1,0)$ & 3 & 2 & 8 \\
\hline $\begin{array}{l}\mathrm{H} 2 \\
\mathrm{H3}\end{array}$ & $\begin{array}{l}(12|13,13,24| 12) \\
(12|13,13,24| 13)\end{array}$ & $\begin{array}{l}G_{1}(1,0,1,0,1,0,1,0) \\
G_{1}(1,0,1,0,1,0,1,0)\end{array}$ & $\begin{array}{l}1 \\
1\end{array}$ & $\begin{array}{l}2 \\
1\end{array}$ & $\begin{array}{l}2 \\
2\end{array}$ \\
\hline I & $(12,13|\varnothing| 12,14)$ & $\begin{array}{c}G_{1}(1,1,1,1,1,1) \\
G_{2}(1,1)\end{array}$ & 0 & 2 & 2 \\
\hline $\mathbf{J}$ & $(12,13|12,12| 14)$ & $\begin{array}{c}G_{1}(1,1,1,0,1,0) \\
G_{2}(1,1)\end{array}$ & 1 & 1 & 4 \\
\hline $\mathbf{K 1}$ & $(12,13|14,14,14,14| \varnothing)$ & $G_{1}(1,1,1,1,1,0,1,0)$ & 3 & 2 & 8 \\
\hline $\mathbf{K} 2$ & $(12,13|14,14| 12)$ & $G_{1}(1,1,1,1,1,0,1,0)$ & 1 & 2 & 2 \\
\hline $\mathbf{K} \mathbf{3}$ & $(12,13|14,14| 14)$ & $G_{1}(1,1,1,1,1,0,1,0)$ & 1 & 1 & 2 \\
\hline $\mathbf{L 1}$ & $(12,13|12,12,12,34| \varnothing)$ & $G_{1}(1,0,1,0,1,0,1,0)$ & 2 & 1 & 4 \\
\hline $\mathbf{L} 2$ & $(12,13|12,34| 12)$ & $G_{1}(1,0,1,0,1,0,1,0)$ & 0 & 1 & 1 \\
\hline $\mathbf{M}$ & $(12,34|\varnothing| 12,13)$ & $\begin{array}{l}G_{1}(1,1,1,1) \\
G_{2}(1,1,1,1)\end{array}$ & 0 & 2 & 2 \\
\hline $\mathbf{N}$ & $(12,34|12,12| 13)$ & $\begin{array}{l}G_{1}(1,1,1,1) \\
G_{2}(1,1,1,1)\end{array}$ & 1 & 1 & 4 \\
\hline 01 & $(12,34|13,13,13,13| \varnothing)$ & $G_{1}(1,1,1,0,-1,1,0)$ & 3 & 2 & 8 \\
\hline 02 & $(12,34|13,13| 12)$ & $G_{1}(1,1,1,0,1,-1,1,0)$ & 1 & 2 & 2 \\
\hline $\mathbf{0 3}$ & $(12,34|13,13| 13)$ & $G_{1}(1,1,1,0,1,-1,1,0)$ & 1 & 1 & 2 \\
\hline P1 & $(12,13,14|12,12,12| \varnothing)$ & $G_{1}(1,1,1,1,1,0,1,0)$ & 2 & 1 & 4 \\
\hline P2 & $(12,13,14|12| 12)$ & $G_{1}(1,1,1,1,1,0,1,0)$ & 0 & 1 & 1 \\
\hline Q1 & $(12,13,14|13,13,13| \varnothing)$ & $G_{1}(1,1,1,0,1,1,1,0)$ & 2 & 1 & 4 \\
\hline $\mathbf{Q 2}$ & $(12,13,14|13| 12)$ & $G_{1}(1,1,1,0,1,1,1,0)$ & 0 & 1 & 1 \\
\hline
\end{tabular}




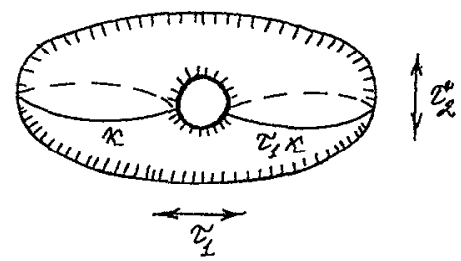

FIG. 7. $\tau_{2}$-oval.

The basis $(a, b)=\left(a_{1}, \ldots, a_{9}, b_{1}, \ldots, b_{9}\right)$ in $H_{1}(\Gamma)$ is called $\tau_{1}$-basis if

$$
\begin{gathered}
\tau_{1}\left(a_{1}\right)=a_{7}, \tau_{1}\left(a_{2}\right)=a_{8}, \tau_{1}\left(a_{3}\right)=a_{9}, \tau_{1}\left(a_{4}\right)=a_{4}, \tau_{1}\left(a_{5}\right)=a_{5}, \tau_{1}\left(a_{6}\right)=a_{6}, \\
\tau_{1}\left(a_{7}\right)=a_{1}, \tau_{1}\left(a_{8}\right)=a_{2}, \tau_{1}\left(a_{9}\right)=a_{3}, \tau_{1}\left(b_{1}\right)=-b_{7}, \tau_{1}\left(b_{2}\right)=-b_{8}, \tau_{1}\left(b_{3}\right)=-b_{9}, \\
\tau_{1}\left(b_{4}\right)=-a_{4}, \tau_{1}\left(b_{5}\right)=-b_{5}, \tau_{1}\left(b_{6}\right)=-b_{6}, \tau_{1}\left(b_{7}\right)=-b_{1}, \tau_{1}\left(b_{8}\right)=-b_{2}, \tau_{1}\left(b_{9}\right)=-b_{3} .
\end{gathered}
$$

In this basis the real part $T \subset \operatorname{Prym}_{\sigma} \Gamma$ of the Prym variety pick up the form ${ }^{6}$

$$
T=\left\{z_{0} \in \mathbf{C}^{9}: \tau_{1} z_{0}=-z_{0}, \sigma z_{0} \equiv-z_{0}+\sigma \Delta-\Delta(\bmod \Lambda)\right\},
$$

where $\Lambda=\left\{2 \pi \sqrt{-1} N+M B \mid N, M \in C^{9}\right\}$ is the lattice of the periods.

Reducing the Riemann theta-function to Prym theta-function we get

Theorem 2. The components $W_{j s}$ of the angular velocity of the rotation of a rigid body around a fixed point in a quadratic potential field are equal to

$(* *) W_{j s}(t)=\left(I_{j}-I_{s}\right) \hat{\mu}_{j s} \exp \hat{K}_{j s} \epsilon^{-1}\left(\infty_{i}, \infty_{j}\right)$

$$
\times \frac{\sum_{i=0}^{1} \Theta\left[\begin{array}{ccccccc}
v_{1}^{1}+\frac{i}{2} & v_{1}^{2}+\frac{i}{2} & v_{1}^{3}+\frac{i}{2} & v_{1}^{4} & v_{1}^{5} & v_{1}^{6} \\
v_{2}^{1} & v_{2}^{2} & v_{2}^{3} & v_{2}^{4} & v_{2}^{5} & v_{2}^{6}
\end{array}\right](\hat{z}+t \hat{U} \mid 2 \Pi) \Theta\left[\begin{array}{ccc}
\frac{i}{2} & \frac{i}{2} & \frac{i}{2} \\
0 & 0 & 0
\end{array}\right](\hat{A} \mid 2 \zeta)}{\sum_{i=0}^{1} \Theta\left[\begin{array}{cccccc}
\frac{i}{2} & \frac{i}{2} & \frac{i}{2} & 0 & 0 & 0 \\
0 & 0 & 0 & 0 & 0 & 0
\end{array}\right](\hat{z}+t \hat{U} \mid 2 \Pi) \Theta\left[\begin{array}{ccc}
\frac{i}{2} & \frac{i}{2} & \frac{i}{2} \\
0 & 0 & 0
\end{array}\right](0 \mid 2 \zeta)} .
$$

In this formula $\mu_{j s}$ depends on $z_{0} \in T$ (see Table XIII), $K_{j s}$ depends on the Prym matrix $\Pi=\left(\Pi_{i j}\right)_{i, j=1}^{6}$ (see Table XIV), $\hat{z}$ is the projection of $z_{0} \in T$ on the Prym variety (see Table XV);

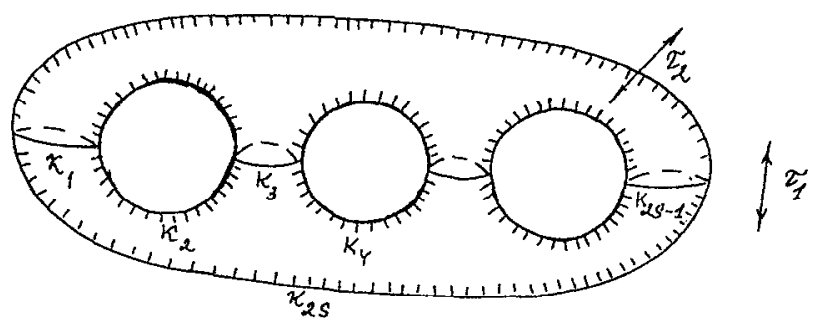

FIG. 8. Garland. 


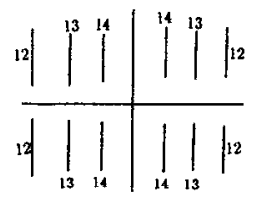

A

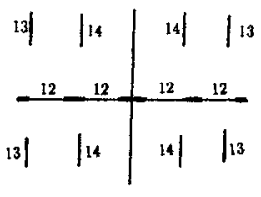

$\mathbf{B 1}$
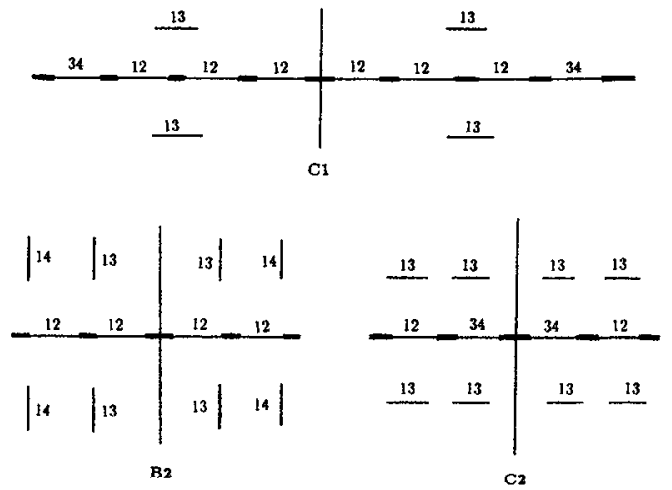

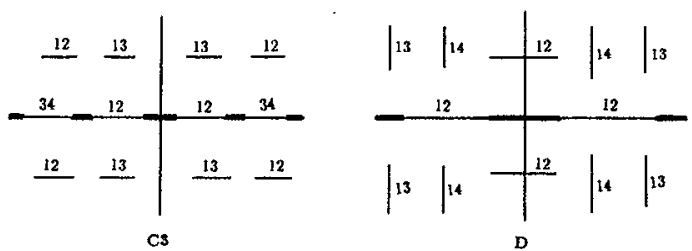

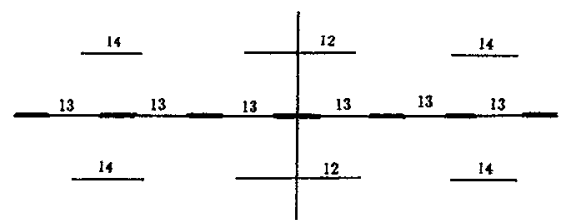

E1

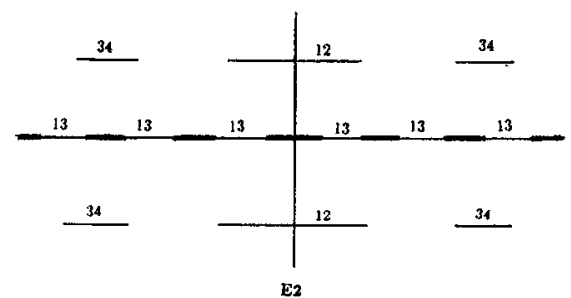

FIG. 9. The different components of the space $H_{4}$. 

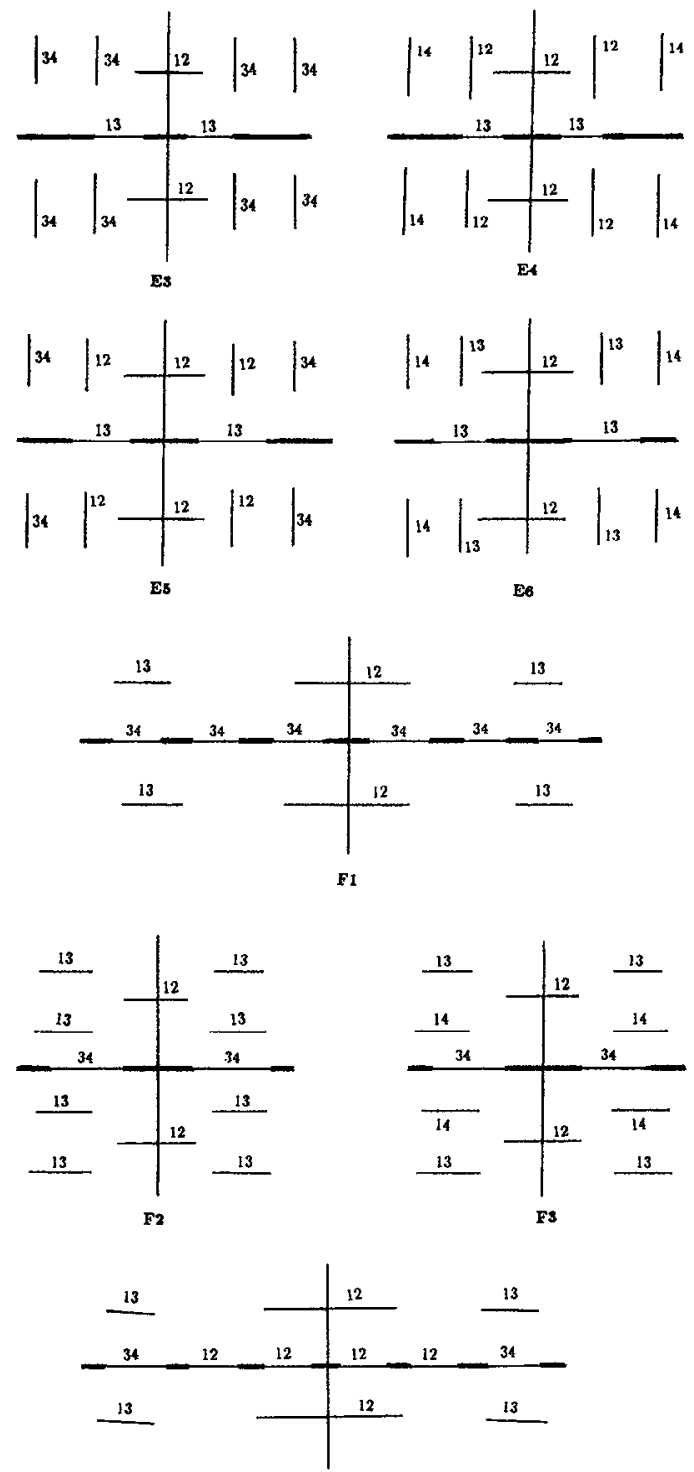

G

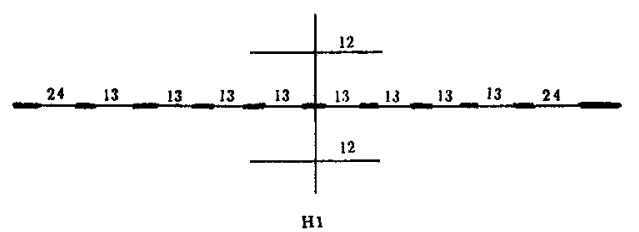

FIG. 9 (Continued.) 

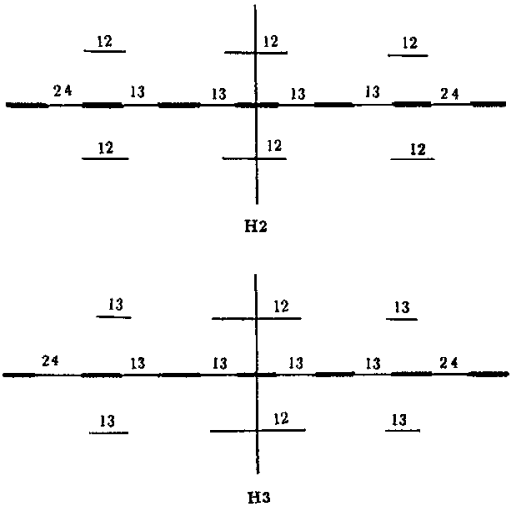

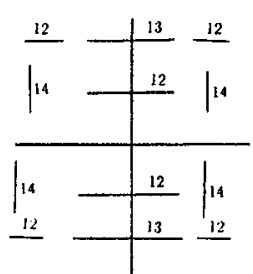

I

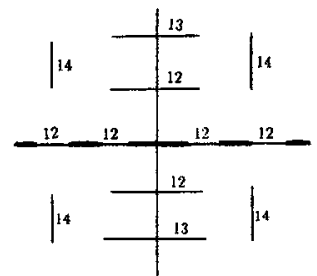

J
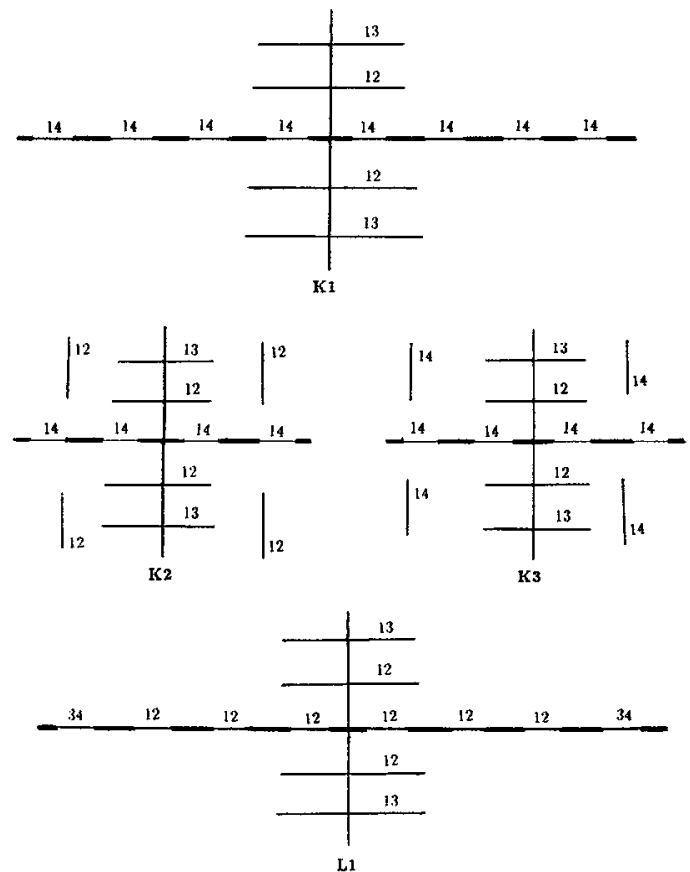

FIG. 9 (Continued.) 

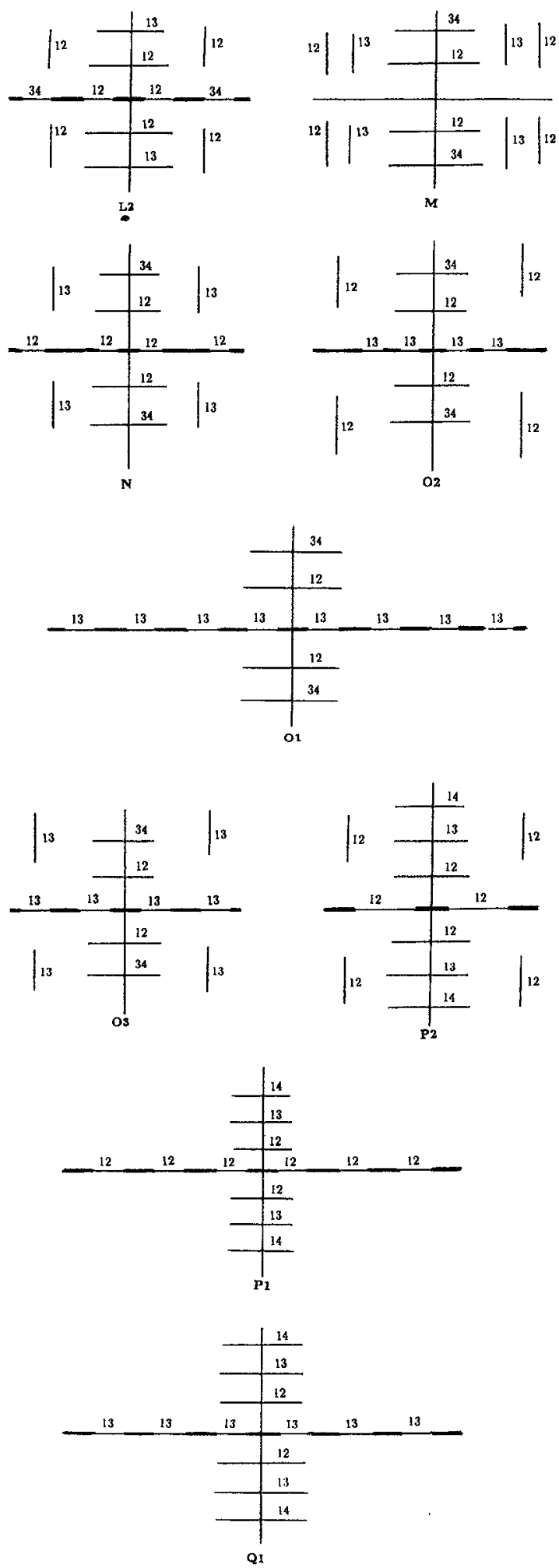

FIG. 9 (Continued.) 


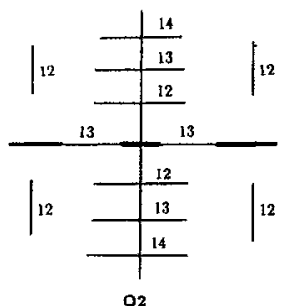

FIG. 9 (Continued.)

$$
\left[\begin{array}{l}
v_{1} \\
v_{2}
\end{array}\right]=\left[\begin{array}{llllll}
v_{1}^{1} & v_{1}^{2} & v_{1}^{3} & v_{1}^{4} & v_{1}^{5} & v_{1}^{6} \\
v_{2}^{1} & v_{2}^{2} & v_{2}^{3} & v_{2}^{4} & v_{2}^{5} & v_{2}^{6}
\end{array}\right]
$$

is a semiperiod (see Table XVI);

$\hat{U}$ is the projection of the vector $U=I_{1} U^{1}+I_{2} U^{2}+I_{3} U^{3}+I_{4} U^{4}$ on the Prym variety, $\hat{A}$ is an integral on $\Gamma / \sigma$

$$
\begin{aligned}
\hat{A} & =\left(\int_{\infty_{s}}^{\infty_{j}} \omega_{1}-\omega_{7}, \int_{\infty_{s}}^{\infty_{j}} \omega_{2}-\omega_{8}, \int_{\infty_{s}}^{\infty_{j}} \omega_{3}-\omega_{9}\right) \\
& =\left(A_{1}\left(\infty_{j}-\infty_{s}\right)-A_{7}\left(\infty_{j}-\infty_{s}\right), A_{2}\left(\infty_{j}-\infty_{s}\right)-A_{8}\left(\infty_{j}-\infty_{s}\right), A_{3}\left(\infty_{j}-\infty_{s}\right)-A_{9}\left(\infty_{j}-\infty_{s}\right)\right) .
\end{aligned}
$$

The Prym-matrix $\Pi, \hat{A}=\hat{A}_{j s}$ and $\hat{U}$ have real symmetries-see Tables XVII-XIX. All tables are for the component $\mathrm{A}$ of the space $\mathrm{H}_{4}$.

Remark: In the other 35 components of the space $H_{4}$ the formula is analogous, but we do not compute the values of the quantities and their symmetries, because of the many computations, which are similar to the above.

Proof: To prove the theorem it is convenient to work with characteristics

$$
z=\left[\begin{array}{l}
\varphi \\
\psi
\end{array}\right]_{B}=2 \pi \sqrt{-1} \psi+B \varphi
$$

Let $\Gamma \in \mathbf{A} \subset H_{4}$. The basis $(a, b)$ in Fig. 10 is a $\sigma$-basis. In this basis $\sigma \Delta=\Delta^{17}$ and

$$
z_{0}=\left[\begin{array}{lllllllll}
\varphi_{1} & \varphi_{2} & \varphi_{3} & \varphi_{4} & \varphi_{5} & \varphi_{6} & \varphi_{1}+n_{1} & \varphi_{2}+n_{2} & \varphi_{3}+n_{3} \\
\psi_{1} & \psi_{2} & \psi_{3} & \psi_{4} & \psi_{5} & \psi_{6} & \psi_{1}+m_{1} & \psi_{2}+m_{2} & \psi_{3}+m_{3}
\end{array}\right]
$$

where $n_{1}, n_{2}, n_{3}, m_{1}, m_{2}, m_{3} \in \mathbf{Z}$.

TABLE XIII. $\bar{\mu}_{j s}$ on the component $A$ of the space $H_{4}$.

\begin{tabular}{ll}
\hline \hline$j=2, s=1$ & $\exp \pi i\left(\varphi_{1}+\varphi_{2}+\varphi_{3}+\psi_{1}+\frac{1}{2}\right)$ \\
$j=3, s=1$ & $\exp \pi i\left(\varphi_{2}+\varphi_{3}+\psi_{2}+\frac{1}{2}\right)$ \\
$j=4, s=1$ & $\exp \pi i\left(\varphi_{3}+\psi_{3}+\frac{1}{2}\right)$ \\
$j=2, s=3$ & $\exp \pi i\left(\varphi_{1}+\psi_{1}-\psi_{2}+\frac{1}{2}\right)$ \\
$j=2, s=4$ & $\exp \pi i\left(\varphi_{1}+\varphi_{2}+\psi_{1}-\psi_{3}+\frac{1}{2}\right)$ \\
$j=3, s=4$ & $\exp \pi i\left(\varphi_{2}+\psi_{2}-\psi_{3}+\frac{1}{2}\right)$ \\
\hline
\end{tabular}


TABLE XIV. $\hat{K}_{j s}$ on the component $A$ of the space $H_{4}$.

\begin{tabular}{lc}
\hline \hline$j=2, s=1$ & $-\frac{\Pi_{44}}{2}$ \\
$j=3, s=1$ & $-\frac{\Pi_{55}}{\Pi_{66}^{2}}$ \\
$j=4, s=1$ & $-\frac{\Pi_{44}-\Pi_{55}}{\Pi_{44}-\Pi_{66}}$ \\
$j=2, s=3$ & $-\frac{\Pi_{55}-\Pi_{66}}{2}$ \\
$j=2, s=4$ & $-\frac{\pi^{2}}{j=3, s=4}$ \\
\hline
\end{tabular}

The basis $\left(a^{\prime}, b^{\prime}\right)=\left(a_{1}, a_{2}, a_{3}, a_{1}-a_{7}, a_{2}-a_{8}, a_{3}-a_{9}, a_{1}-a_{4}, a_{2}-a_{5}, a_{3}-a_{6}, b_{1}+b_{4}\right.$ $\left.+b_{7}, b_{2}+b_{5}+b_{8}, b_{3}+b_{6}+b_{9},-b_{7},-b_{8},-b_{9},-b_{4},-b_{5},-b_{6}\right)$ is a $\tau_{1}$-basis. In this basis the vector $z_{0} \equiv-\sigma z_{0}(\bmod \Lambda)$ has the form

$$
z_{0}^{\prime}=\left[\begin{array}{ccccccccc}
2 \varphi_{1}+\varphi_{4}+n_{1} & 2 \varphi_{2}+\varphi_{5}+n_{2} & \cdots & -\varphi_{1}-n_{1} & -\varphi_{2}-n_{2} & \cdots & -\varphi_{4} & -\varphi_{5} & \cdots \\
\psi_{1} & \psi_{2} & \cdots & -m_{1} & -m_{2} & \cdots & \psi_{1}-\psi_{4} & \psi_{2}-\psi_{5} & \cdots
\end{array}\right],
$$

where $B^{\prime}$ is the Riemann matrix in the basis $\left(a^{\prime}, b^{\prime}\right)$. (See Ref. 18 for the formula, changing characteristics when the basis is changed). If $z=\left[{ }_{\psi}^{\varphi}\right]_{B}, z^{\prime}=\left[\begin{array}{c}\varphi^{\prime} \\ \psi^{\prime}\end{array}\right]_{B^{\prime}}$ where

TABLE XV. $\hat{z}$ on the component $A$ of the space $H_{4}$.

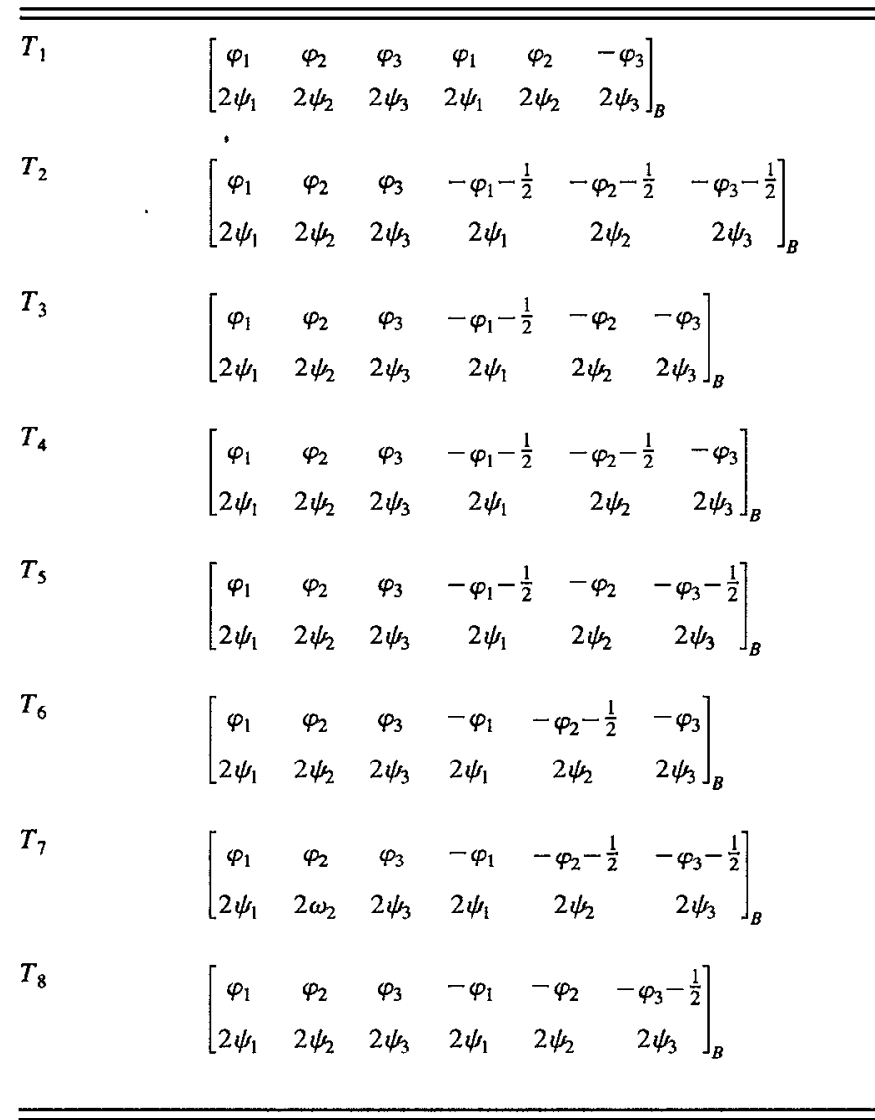


TABLE XVI. The semiperiod on the component $A$ of the space $H_{4}$.

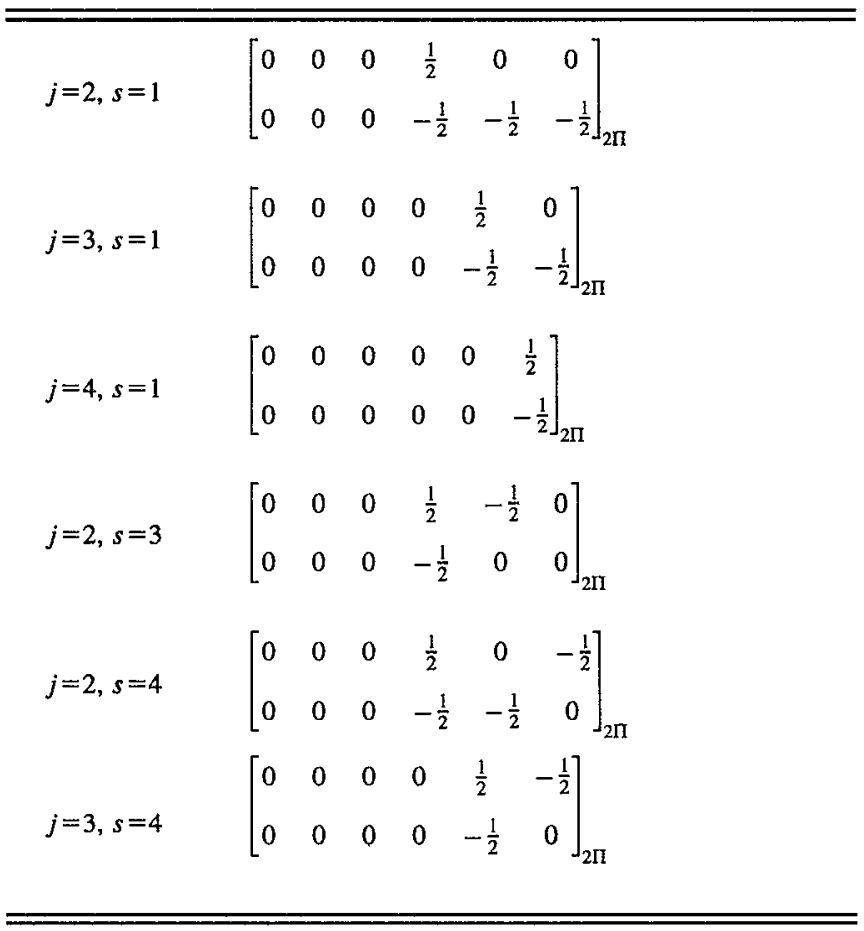

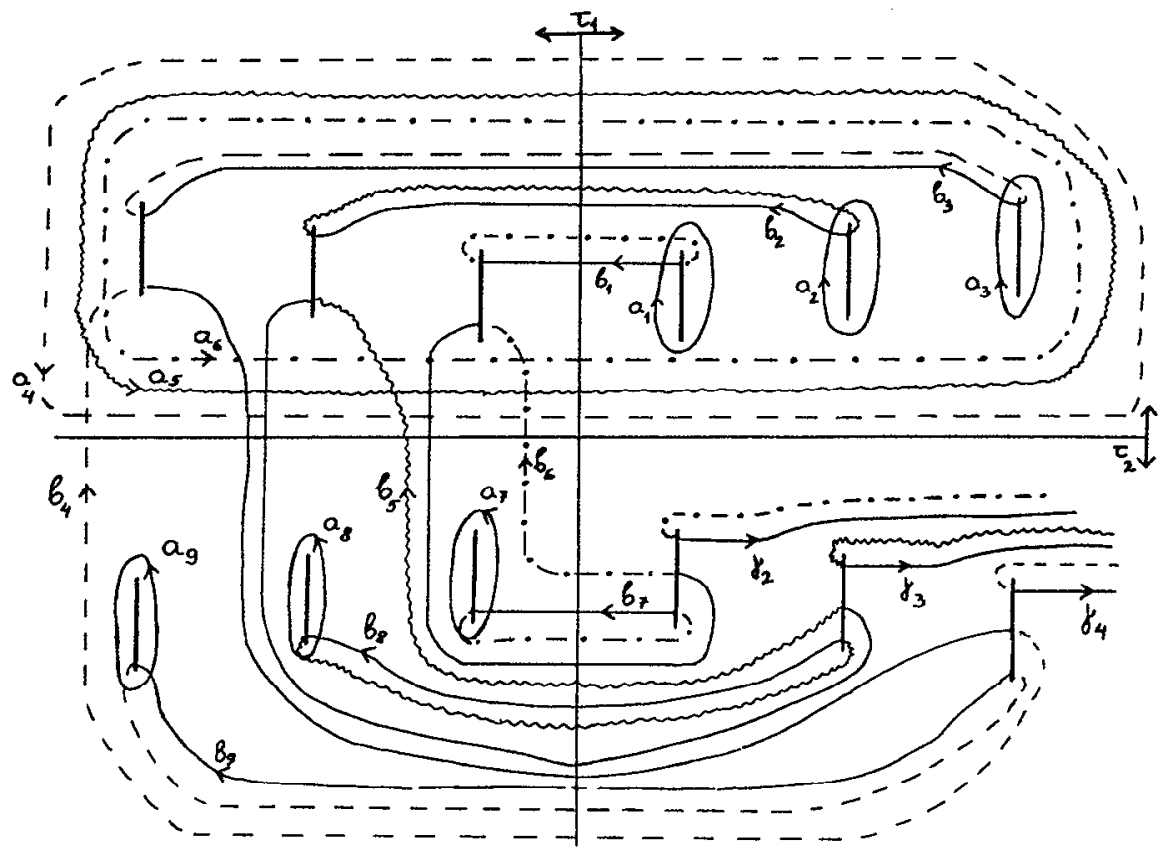

FIG. 10. $\sigma$-basis on the component $\mathbf{A} \subset H_{4}$. 


$$
\begin{aligned}
& \varphi^{\prime}=p \varphi-q \psi \\
& \psi^{\prime}=s \psi-r \varphi
\end{aligned} \text { and }\left(\begin{array}{l}
a^{\prime} \\
b^{\prime}
\end{array}\right)=\left(\begin{array}{ll}
p & q \\
r & s
\end{array}\right)\left(\begin{array}{l}
a \\
b
\end{array}\right),\left(\begin{array}{ll}
p & q \\
r & s
\end{array}\right) \in S p(g, Z)
$$

By the condition $\tau_{1} z_{0}^{\prime}=-z_{0}^{\prime}$ we find that $m_{1}=m_{2}=m_{3}=0 ; \psi_{4}=2 \psi_{1}, \psi_{5}=2 \psi_{2}, \psi_{6}=2 \psi_{3}$, $\psi_{4}=-\psi_{1}-n_{1} / 2, \psi_{5}=-\psi_{2}-n_{2} / 2, \psi_{6}=-\psi_{3}-n_{3} / 2$. Therefore

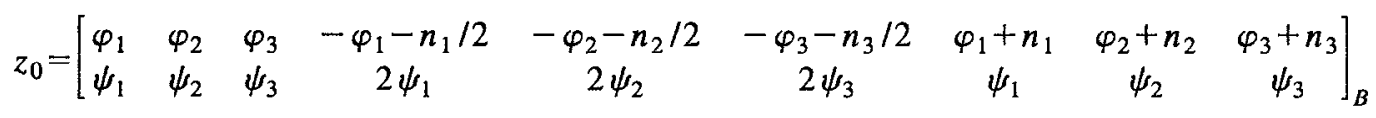

and the variety $T=\left\{z_{0}: \tau_{1} z_{0}=-z_{0}, \sigma z_{0} \equiv-z_{0}(\bmod \Lambda)\right\} \subset J(\Gamma)$ consists of 8 components $T_{1}, \ldots, T_{8}$, where

$$
\begin{aligned}
T_{1}= & \left\{z_{0}=2 \pi \sqrt{-1}\left(\psi_{1}, \psi_{2}, \psi_{3}, 2 \psi_{1}, 2 \psi_{2}, 2 \psi_{3}, \psi_{1}, \psi_{2}, \psi_{3}\right)\right. \\
& \left.+\left(\varphi_{1}, \varphi_{2}, \varphi_{3},-\varphi_{1},-\varphi_{2},-\varphi_{3}, \varphi_{1}, \varphi_{2}, \varphi_{3}\right) B\right\} \\
T_{2}=\left\{z_{0}=2 \pi \sqrt{-1}\left(\psi_{1}, \psi_{2}, \psi_{3}, 2 \psi_{1}, 2 \psi_{2}, 2 \psi_{3}, \psi_{1}, \psi_{2}, \psi_{3}\right)\right. & \left.+\left(\varphi_{1}, \varphi_{2}, \varphi_{3},-\varphi_{1}-\frac{1}{2},-\varphi_{2}-\frac{1}{2},-\varphi_{3}-\frac{1}{2}, \varphi_{1}, \varphi_{2}, \varphi_{3}\right) B\right\} \\
T_{3}= & \left\{z_{0}=2 \pi \sqrt{-1}\left(\psi_{1}, \psi_{2}, \psi_{3}, 2 \psi_{1}, 2 \psi_{2}, 2 \psi_{3}, \psi_{1}, \psi_{2}, \psi_{3}\right)\right. \\
& \left.+\left(\varphi_{1}, \varphi_{2}, \varphi_{3},-\varphi_{1}-\frac{1}{2},-\varphi_{2},-\varphi_{3}, \varphi_{1}, \varphi_{2}, \varphi_{3}\right) B\right\} \\
T_{4}= & \left\{z_{0}=2 \pi \sqrt{-1}\left(\psi_{1}, \psi_{2}, \psi_{3}, 2 \psi_{1}, 2 \psi_{2}, 2 \psi_{3}, \psi_{1}, \psi_{2}, \psi_{3}\right)\right. \\
& \left.+\left(\varphi_{1}, \varphi_{2}, \varphi_{3},-\varphi_{1}-\frac{1}{2},-\varphi_{2}-\frac{1}{2},-\varphi_{3}, \varphi_{1}, \varphi_{2}, \varphi_{3}\right) B\right\} \\
T_{5}= & \left\{z_{0}=2 \pi \sqrt{-1}\left(\psi_{1}, \psi_{2}, \psi_{3}, 2 \psi_{1}, 2 \psi_{2}, 2 \psi_{3}, \psi_{1}, \psi_{2}, \psi_{3}\right)\right. \\
& \left.+\left(\varphi_{1}, \varphi_{2}, \varphi_{3},-\varphi_{1}-\frac{1}{2},-\varphi_{2},-\varphi_{3}-\frac{1}{2}, \varphi_{1}, \varphi_{2}, \varphi_{3}\right) B\right\} \\
T_{8}=\{ & \left\{z_{0}=2 \pi \sqrt{-1}\left(\psi_{1}, \psi_{2}, \psi_{3}, 2 \psi_{1}, 2 \psi_{2}, 2 \psi_{3}, \psi_{1}, \psi_{2}, \psi_{3}\right)\right. \\
& \left.+\left(\varphi_{1}, \varphi_{2}, \varphi_{3},-\varphi_{1},-\varphi_{2},-\varphi_{3}-\frac{1}{2}, \varphi_{1}, \varphi_{2}, \varphi_{3}\right) B\right\} \\
T_{6}=\{ & \left\{z_{0}=2 \pi \sqrt{-1}\left(\psi_{1}, \psi_{2}, \psi_{3}, 2 \psi_{1}, 2 \psi_{2}, 2 \psi_{3}, \psi_{1}, \psi_{2}, \psi_{3}\right)\right. \\
& \left.+\left(\varphi_{1}, \varphi_{2}, \varphi_{3},-\varphi_{1},-\varphi_{2}-\frac{1}{2},-\varphi_{3}, \varphi_{1}, \varphi_{2}, \varphi_{3}\right) B\right\} \\
T_{7}= & \left\{z_{0}=2 \pi \sqrt{-1}\left(\psi_{1}, \psi_{2}, \psi_{3}, 2 \psi_{1}, 2 \psi_{2}, 2 \psi_{3}, \psi_{1}, \psi_{2}, \psi_{3}\right)\right. \\
& \left.+\left(\varphi_{1}, \varphi_{2}, \varphi_{3},-\varphi_{1},-\varphi_{2}-\frac{1}{2},-\varphi_{3}-\frac{1}{2}, \varphi_{1}, \varphi_{2}, \varphi_{3}\right) B\right\} \\
& \\
& \\
&
\end{aligned}
$$

Every component is a six-dimensional real torus, the coordinates of which are the real numbers $\varphi_{1}, \varphi_{2}, \varphi_{3}, \psi_{1}, \psi_{2}, \psi_{3} \in \mathbf{S}^{1}=\mathbf{R}_{/ \mathbf{z}}$. Thus we obtain 


$$
\hat{z}_{0}=\left(\left(z_{0}\right)_{1}+\left(z_{0}\right)_{7},\left(z_{0}\right)_{2}+\left(z_{0}\right)_{8},\left(z_{0}\right)_{3}+\left(z_{0}\right)_{9},\left(z_{0}\right)_{4},\left(z_{0}\right)_{5},\left(z_{0}\right)_{6}\right)
$$

see Table XV. Let

$$
\begin{gathered}
\hat{I}_{j s}=\int_{\infty_{s}}^{\infty_{j}} \sum_{i=1}^{4} I_{i} \Omega_{i}, \quad j, s=1, \ldots, 4, \\
\hat{A}_{j}=\left(A_{1}\left(\infty_{j}\right)+A_{7}\left(\infty_{j}\right), A_{2}\left(\infty_{j}\right)+A_{8}\left(\infty_{j}\right), A_{3}\left(\infty_{j}\right)+A_{9}\left(\infty_{j}\right), A_{4}\left(\infty_{j}\right), A_{5}\left(\infty_{j}\right), A_{6}\left(\infty_{j}\right)\right), \\
\hat{A}_{j s}=\hat{A}_{j}-\hat{A}_{s}, \quad j, s=1, \ldots, 4, \\
\hat{A}_{j}=\left(A_{1}\left(\infty_{j}\right)-A_{7}\left(\infty_{j}\right), A_{2}\left(\infty_{j}\right)-A_{8}\left(\infty_{j}\right), A_{3}\left(\infty_{j}\right)-A_{9}\left(\infty_{j}\right)\right), \\
\hat{A}_{j s}=\hat{A}_{j}-\hat{A}_{s}, \quad j, s=1, \ldots, 4 .
\end{gathered}
$$

Using the formula

$$
(*) \Theta\left[\begin{array}{l}
\alpha \\
\beta
\end{array}\right](z \mid B)=\exp \left\{\frac{1}{2}\langle B \alpha, \alpha\rangle+\langle z+2 \pi \sqrt{-1} \beta, \alpha\rangle\right\} \cdot \Theta(z+2 \pi \sqrt{-1} \beta+B \alpha)
$$

we determine that the denominator of $(* *)$ is

$$
\sum_{i=0}^{1} \Theta\left[\begin{array}{cccccc}
\frac{i}{2} & \frac{i}{2} & \frac{i}{2} & 0 & 0 & 0 \\
0 & 0 & 0 & 0 & 0 & 0
\end{array}\right](\hat{z}+t \hat{U} \mid 2 \Pi) \cdot \Theta\left[\begin{array}{ccc}
\frac{i}{2} & \frac{i}{2} & \frac{i}{2} \\
0 & 0 & 0
\end{array}\right](0 \mid 2 \zeta)
$$

and that

$$
\Theta\left(A_{j s}+z_{0}+t U\right)=\sum_{i=0}^{1} \Theta\left[\begin{array}{cccccc}
\frac{i}{2} & \frac{i}{2} & \frac{i}{2} & 0 & 0 & 0 \\
0 & 0 & 0 & 0 & 0 & 0
\end{array}\right]\left(\hat{A}_{j s}+\hat{z}+t \hat{U} \mid 2 \Pi\right) \cdot \Theta\left[\begin{array}{ccc}
\frac{i}{2} & \frac{i}{2} & \frac{i}{2} \\
0 & 0 & 0
\end{array}\right]\left(\hat{A}_{j s} \mid 2 \zeta\right)
$$

Let $j=2, s=1,\left(\Gamma, \tau_{1}, \tau_{2}, \lambda\right) \in \mathbf{A} \subset H_{4}$. Then

$$
\begin{gathered}
\hat{A}_{21}=\left[\begin{array}{cccccc}
0 & 0 & 0 & \frac{1}{2} & 0 & 0 \\
0 & 0 & 0 & -\frac{1}{2} & -\frac{1}{2} & -\frac{1}{2}
\end{array}\right]_{2 \Pi}, \\
I_{21}=\frac{1}{2} \sum_{i=1}^{6} I_{4} U_{4}^{i}=\frac{1}{2} \hat{U}_{4}, \quad \hat{U}=\left(\hat{U}_{1}, \ldots, \hat{U}_{6}\right) .
\end{gathered}
$$

Using the formula $\left(^{*}\right)$ we obtain 


$$
\begin{aligned}
& \Theta\left(\hat{A}_{21}+\hat{z}+t \hat{U} \mid 2 \Pi\right) \cdot \exp \left(t \hat{I}_{21}\right)=\Theta\left(\hat{z}+t \hat{U}+\left[\begin{array}{cccccc}
0 & 0 & 0 & \frac{1}{2} & 0 & 0 \\
0 & 0 & 0 & -\frac{1}{2} & -\frac{1}{2} & -\frac{1}{2}
\end{array}\right]_{2 \Pi} \mid 2 \Pi\right) \cdot \exp \frac{t \hat{U}_{4}}{2} \\
& =\Theta\left[\begin{array}{cccccc}
0 & 0 & 0 & \frac{1}{2} & 0 & 0 \\
0 & 0 & 0 & -\frac{1}{2} & -\frac{1}{2} & -\frac{1}{2}
\end{array}\right](\hat{z}+t \hat{U} \mid 2 \Pi) \\
& \times \exp \frac{t \hat{U}_{4}}{2} \cdot \exp \left[-\frac{\Pi_{44}}{4}+\frac{\pi \sqrt{-1}}{2}-\frac{\hat{z}_{4}+t \hat{U}_{4}}{2}\right] \\
& =\Theta\left[\begin{array}{cccccc}
0 & 0 & 0 & \frac{1}{2} & 0 & 0 \\
0 & 0 & 0 & -\frac{1}{2} & -\frac{1}{2} & -\frac{1}{2}
\end{array}\right](\hat{z}+t \hat{U} \mid 2 \Pi) \\
& \cdot \exp \left[-\frac{\Pi_{44}}{4}-\frac{\hat{z}_{4}}{2}+\frac{\pi \sqrt{-1}}{2}\right]
\end{aligned}
$$

where $\hat{z}=\left(\hat{z}_{1}, \hat{z}_{2}, \hat{z}_{3}, \hat{z}_{4}, \hat{z}_{5}, \hat{z}_{6}\right)$. Further,

$$
\begin{aligned}
& \Theta\left[\begin{array}{cccccc}
\frac{1}{2} & \frac{1}{2} & \frac{1}{2} & 0 & 0 & 0 \\
0 & 0 & 0 & 0 & 0 & 0
\end{array}\right]\left(\hat{A}_{21}+\hat{z}+t \hat{U} \mid 2 \Pi\right) \cdot \exp \left(t \hat{I}_{21}\right) \\
& =\Theta\left(\left[\begin{array}{cccccc}
\frac{1}{2} & \frac{1}{2} & \frac{1}{2} & 0 & 0 & 0 \\
0 & 0 & 0 & 0 & 0 & 0
\end{array}\right]_{2 \Pi}+\hat{A}_{21}+\hat{z}+t \hat{U} \mid 2 \Pi\right) \\
& \cdot \exp \left(t \hat{I}_{21}\right) \exp \left(\frac{\Pi_{11}+\Pi_{22}+\Pi_{33}}{4}+\frac{\Pi_{12}+\Pi_{13}+\Pi_{23}}{2}+\frac{\left(\hat{A}_{21}\right)_{1}+\left(\hat{A}_{21}\right)_{2}+\left(\hat{A}_{21}\right)_{3}}{2}\right. \\
& \left.+\frac{t \hat{U}_{1}+t \hat{U}_{2}+t \hat{U}_{3}}{2}+\frac{\hat{z}_{1}+\hat{z}_{2}+\hat{z}_{3}}{2}\right) \\
& =\Theta\left(\left[\begin{array}{cccccc}
\frac{1}{2} & \frac{1}{2} & \frac{1}{2} & 0 & 0 & 0 \\
0 & 0 & 0 & 0 & 0 & 0
\end{array}\right]_{2 \Pi}+\left[\begin{array}{cccccc}
0 & 0 & 0 & \frac{1}{2} & 0 & 0 \\
0 & 0 & 0 & -\frac{1}{2} & -\frac{1}{2} & -\frac{1}{2}
\end{array}\right]_{2 \Pi}+\hat{z}+t \hat{U} \mid 2 \Pi\right) \\
& \times \exp \frac{t \hat{U}_{4}}{2} \exp \left(\frac{\Pi_{11}+\Pi_{22}+\Pi_{33}}{4}+\frac{\Pi_{12}+\Pi_{13}+\Pi_{23}}{2}+\frac{t \hat{U}_{1}+t \hat{U}_{2}+t \hat{U}_{3}}{2}\right. \\
& \left.+\frac{\Pi_{14}+\Pi_{24}+\Pi_{34}}{2}+\frac{\hat{z}_{1}+\hat{z}_{2}+\hat{z}_{3}}{2}\right) \\
& =\Theta\left([]^{\frac{1}{2}} \hat{z}+t \hat{U} \mid 2 \Pi\right) \exp \left(\frac{\Pi_{11}+\Pi_{22}+\Pi_{33}+\Pi_{44}}{4}-\frac{\Pi_{12}+\Pi_{13}+\Pi_{14}+\Pi_{23}+\Pi_{24}+\Pi_{34}}{2}\right.
\end{aligned}
$$




$$
\begin{aligned}
& -\frac{t \hat{U}_{1}+t \hat{U}_{2}+t \hat{U}_{3}+t \hat{U}_{4}}{2}-\frac{\hat{z}_{1}+\hat{z}_{2}+\hat{z}_{3}+\hat{z}_{4}}{2} \\
& +\frac{\pi \sqrt{-1}}{2}+\frac{\Pi_{11}+\Pi_{22}+\Pi_{33}}{4}+\frac{\Pi_{12}+\Pi_{13}+\Pi_{23}}{2}+\frac{\hat{z}_{1}+\hat{z}_{2}+\hat{z}_{3}}{2}+\frac{t \hat{U}_{4}}{2} \\
& \left.+\frac{t \hat{U}_{1}+t \hat{U}_{2}+t \hat{U}_{3}}{2}+\frac{\Pi_{14}+\Pi_{24}+\Pi_{34}}{2}\right) \\
& =\Theta\left(\left[\begin{array}{cccccc}
0 & 0 & 0 & \frac{1}{2} & 0 & 0 \\
0 & 0 & 0 & -\frac{1}{2} & -\frac{1}{2} & -\frac{1}{2}
\end{array}\right]+\hat{z}+t \hat{U} \mid 2 \Pi\right) \cdot \exp \left(-\frac{\Pi_{44}}{4}-\frac{\hat{z}_{4}}{2}+\frac{\pi \sqrt{-1}}{2}\right), \\
& \hat{K}_{21}=-\frac{\Pi_{44}}{4}, \\
& \hat{\mu}_{21}=\exp \left[\pi \sqrt{-1}\left(\varphi_{1}+\varphi_{2}+\varphi_{3}+\psi_{3}+\frac{1}{2}\right)\right] .
\end{aligned}
$$

We compute that

$$
\mu_{2}=\mu_{1} \cdot \exp \left(\pi \sqrt{-1} .3 \psi_{1}\right)
$$

and

$$
\exp \left\langle\varphi\left(z_{0}\right), A_{21}\right\rangle=\exp \left[\pi \sqrt{-1}\left(\varphi_{1}+\varphi_{2}+\varphi_{3}-2 \psi_{1}\right)+\frac{\hat{z}_{4}}{2}\right]
$$

The received expressions, substituted in $\left(^{* *}\right)$ get us $W_{21}$ for the component $\mathbf{A}$ of the space $H_{4}$. In the others cases for $j, s=1, \ldots, 4$ the computations are analogous.

The real symmetries of the Prym matrix follow from:

(1) The condition of realness $\bar{B}^{\prime}=P B P$, where

$$
P=\left(\begin{array}{ccc}
0 & 0 & I \\
0 & I & 0 \\
I & 0 & 0
\end{array}\right) ; \quad I=\left(\begin{array}{ccc}
1 & 0 & 0 \\
0 & 1 & 0 \\
0 & 0 & 1
\end{array}\right)
$$

(2) The relations between $B, \Pi$, and $\zeta$.

(3) The formula $B^{\prime}=M^{t} B M^{-1}$ (Ref. 18) for the basis change.

Let the paths $\gamma_{2}, \gamma_{3}, \gamma_{4}$ joint $P_{0}=\infty_{1}$ with $\infty_{2}, \infty_{3}, \infty_{4}$ respectively. As we see in Fig. 15

TABLE XVII. The symmetries of $\hat{U}^{s}$ on the component $A$ of the space $H_{4}$.

\begin{tabular}{cc}
\hline Reals & $U_{1}^{s}, U_{2}^{s}, U_{3}^{s}, U_{7}^{s}, U_{8}^{s}, U_{9}^{s}$ \\
\hline & $2 \operatorname{Re} U_{4}^{s}=U_{1}^{s}+U_{7}^{s}$ \\
Other & $2 \operatorname{Re} U_{5}^{s}=U_{2}^{s}+U_{8}^{s}$ \\
symmetries & $2 \operatorname{Re} U_{6}^{s}=U_{3}^{s}+U_{9}^{s}$ \\
\hline
\end{tabular}


TABLE XVIII. The symmetries of $\hat{\hat{A}}_{j s}$ on the component $A$ of the space $H_{4}$.

\begin{tabular}{lll}
\hline \hline$j=2, s=1$ & $\operatorname{Re}$ & $\hat{\hat{A}}_{21}=\left(\frac{\zeta_{11}}{2}, \frac{\zeta_{12}}{2}, \frac{\zeta_{13}}{2}\right)$ \\
$j=3, s=1$ & $\operatorname{Re}$ & $\hat{\hat{A}}_{31}=\left(\frac{\zeta_{21}}{2}, \frac{\zeta_{22}}{2}, \frac{\zeta_{23}}{2}\right)$ \\
$j=4, s=1$ & $\operatorname{Re}$ & $\hat{\hat{A}}_{41}=\left(\frac{\zeta_{31}}{2}, \frac{\zeta_{32}}{2}, \frac{\zeta_{33}}{2}\right)$ \\
$j=2, s=3$ & $\operatorname{Re}$ & $\hat{\hat{A}}_{23}=\left(\frac{\zeta_{11}-\zeta_{21}}{2}, \frac{\zeta_{12}-\zeta_{22}}{2}, \frac{\zeta_{13}-\zeta_{23}}{2}\right)$ \\
$j=2, s=4$ & $\operatorname{Re}$ & $\hat{\hat{A}}_{24}=\left(\frac{\zeta_{11}-\zeta_{31}}{2}, \frac{\zeta_{12}-\zeta_{32}}{2}, \frac{\zeta_{13}-\zeta_{33}}{2}\right)$ \\
$j=3, s=4$ & $\operatorname{Re}$ & $\hat{\hat{A}}_{34}=\left(\frac{\zeta_{21}-\zeta_{31}}{2}, \frac{\zeta_{22}-\zeta_{32}}{2}, \frac{\zeta_{23}-\zeta_{33}}{2}\right)$ \\
& &
\end{tabular}

$$
\begin{gathered}
\sigma \gamma_{2}-\gamma_{2}=-b_{4}+a_{4}+a_{5}+a_{6} \\
\sigma \gamma_{3}-\gamma_{3}=-b_{5}+a_{5}+a_{6} \\
\sigma \gamma_{4}-\gamma_{4}=-b_{6}+a_{6}
\end{gathered}
$$

Using $\sigma^{*} \omega=-\omega$ and $\sigma^{*} \Omega_{s}=\Omega_{s}, \tau_{1}^{*} \Omega_{s}=-\bar{\Omega}_{s}, s=1, \ldots, 4$ we obtain the symmetries of the

$$
\hat{U}^{s}=\left(U_{1}^{s}+U_{7}^{s}, U_{2}^{s}+U_{8}^{s}, U_{3}^{s}+U_{9}^{s}, U_{4}^{s}, U_{5}^{s}, U_{6}^{s}\right)
$$

as well in Table XVII, and of the $\hat{A}_{j s}$ as well in Tables XVIII and XIX.

In the other cases for $j, s=1, \ldots, 4$ the computations are completely analogous. The theorem is proven.

TABLE XIX. The symmetries of the Prym matrix $\Pi$ on the component $A$ of the space $H_{4}$.

\begin{tabular}{ll}
\hline \hline Reals & $\Pi_{i j} ; i=1,2,3 ; j=1, \ldots, 6$ \\
Pure imaginaries & $\Pi_{i j} ; i, j=4,5,6$ \\
& $\operatorname{Re} \Pi_{41}=-\frac{\Pi_{11}}{2}, \operatorname{Re} \Pi_{52}=-\frac{\Pi_{22}}{2}, \operatorname{Re} \Pi_{63}=-\frac{\Pi_{33}}{2}$ \\
& $\operatorname{Re} \Pi_{42}=\operatorname{Re} \Pi_{51}=-\frac{\Pi_{12}}{2}$ \\
Other & $\operatorname{Re} \Pi_{43}=\operatorname{Re} \Pi_{61}=-\frac{\Pi_{13}}{2}$ \\
symmetries & $\operatorname{Re} \Pi_{53}=\operatorname{Re} \Pi_{62}=-\frac{\Pi_{23}}{2}$ \\
& $\Pi_{44}=\operatorname{Im} \Pi_{41}, \Pi_{55}=\operatorname{Im} \Pi_{52}, \Pi_{66}=\operatorname{Im} \Pi_{63}$ \\
& $\Pi_{45}=\frac{1}{2} \operatorname{Im}\left(\Pi_{42}+\Pi_{51}\right)$ \\
& $\Pi_{46}=\frac{1}{2} \operatorname{Im}\left(\Pi_{43}+\Pi_{61}\right)$ \\
& $\Pi_{56}=\frac{1}{2} \operatorname{Im}\left(\Pi_{53}+\Pi_{62}\right)$ \\
\hline
\end{tabular}




\section{ACKNOWLEDGMENT}

This work is supported by Grant No MM-402/94 of NF "Scientific Research."

'B. A. Dubrovin, V. B. Matveev, and S. P. Novikov, "Nonlinear equations of Korteweg-de Vries type, finite-band linear operators and Abelian varieties," Russ. Math. Surv. 31, 55-136 (1976).

${ }^{2}$ A. R. Its, "Inversion of hypereliptic integrals and integration of non-linear differential equations," Vest. Leningr. Univ. Ser. Mat., Mekh. Astr. 7, 39-46 (1976).

${ }^{3}$ I. M. Kricever, "Methods of algebraic geometry in the theory of non-linear equations," Russ. Math. Surv. 32, 185-213 (1977).

${ }^{4} \mathrm{H}$. Mc Kean and P. van Moerbeke, "The spectrum of Hill's operator," Invent. Math. 30, 217-274 (1975).

${ }^{5}$ B. A. Dubrovin and S. M. Natanzon, "Real two-zone solutions of the sine-Gordon equation," Funct. Anal. Prilozh. 16, 27-43 (1982).

${ }^{6}$ B. A. Dubrovin, "Matrix finite-zone operators," Itogi Nauki Tekh. Ser. Sovrem. Probl. Mat. 23, 33-78 (1983) (Russian).

${ }^{7}$ V. E. Zakharov, S. V. Manakov, S. P. Novikov, and L. P. Pitaevskij, Soliton Theory (Nauka, Moscow, 1980) (Russian).

${ }^{8} \mathrm{I}$. V. Cherednic, "On the regularity of "finite-zone" solutions of integrable matrix differential equations," Docl. Acad. Nauk SSSR 266, 593-597 (1982).

${ }^{9}$ A. I. Zhivkov, "Matrix finite-gap potentials with one and two involutions," Bull. Sci. Math. (in press).

${ }^{10} \mathrm{O}$. I. Bogoyavlenskij, "Integrable Euler equations on Lie algebras arising in the problems of mathematical physics," Izv. Acad. Nauk SSSR Ser. Math. 48, 883-938 (1984).

${ }^{11}$ A. I. Zhivkov, "Rotation of a rigid body around a fixed point in a quadratic potential field," Izv. Acad. Nauk SSSR Ser. Math. 54, 879-893 (1990).

${ }^{12}$ V. I. Arnold, "Mathematical methods of the classical mechanics," Springer Graduate Texts Math. 60 (1978).

${ }^{13}$ A. Clebsch, Zur theoric der Riemann Flascher. Math. Ann. 6, 1-15 (1883).

${ }^{14} \mathrm{~S}$. M. Natanzon, "Topological classification of pairs of commuting antiholomorphic involutions of Riemann surfaces," Russ. Math. Surv. 45, 159-160 (1986).

${ }^{15}$ S. M. Natanzon, "Real meromorphic functions on real algebraic curves," Docl. Acad. Nauk SSSR 279, 40-43 (1984) (Russian).

${ }^{16}$ A. I. Zhivkov, "Isospecral classes of matrix finite-zone operators with symmetries," Russ. Math. Surv. 44, I, 197 (1986).

$17 \mathrm{~J}$. Fay, Theta Functions on Riemann Surfaces, Lecture Notes Mathematics, Vol. 352 (Springer, Berlin, 1973).

${ }^{18} \mathrm{~J}$. Igusa, Theta functions (Springer, Berlin, 1972). 